\title{
Jets in Hydrogen-poor Superluminous Supernovae: Constraints from a Comprehensive Analysis of Radio Observations
}

\author{
D. L. Coppejans ${ }^{1}$ (D), R. Margutti ${ }^{1}$ (D) C. Guidorzi ${ }^{2}$ (D) L. Chomiuk $^{3}$ (D), K. D. Alexander ${ }^{4}$ (D), E. Berger ${ }^{4}$ (D), M. F. Bietenholz ${ }^{5,6}$ (D), \\ P. K. Blanchard ${ }^{4}$, P. Challis ${ }^{4}$, R. Chornock ${ }^{7}$, M. Drout ${ }^{8}$, W. Fong ${ }^{1,13}$ (D), A. MacFadyen ${ }^{9}$ (D), G. Migliori ${ }^{10}$ (DD, D. Milisavljevic ${ }^{11}$ (D), \\ M. Nicholl ${ }^{4}$ (D), J. T. Parrent ${ }^{4}$ (D), G. Terreran ${ }^{1}$, and B. A. Zauderer ${ }^{9,12}$ \\ ${ }^{1}$ Center for Interdisciplinary Exploration and Research in Astrophysics (CIERA) and Department of Physics and Astronomy, Northwestern University, Evanston, IL \\ 60208, USA \\ ${ }^{2}$ Department of Physics and Earth Science, University of Ferrara, via Saragat 1, I-44122, Ferrara, Italy \\ ${ }^{3}$ Department of Physics and Astronomy, Michigan State University, East Lansing, MI 48824, USA \\ ${ }^{4}$ Harvard-Smithsonian Center for Astrophysics, 60 Garden St., Cambridge, MA 02138, USA \\ ${ }^{5}$ Hartebeesthoek Radio Observatory, P.O. Box 443, Krugersdorp, 1740, South Africa \\ ${ }^{6}$ Department of Physics and Astronomy, York University, Toronto, M3J 1P3, ON, Canada \\ ${ }^{7}$ Astrophysical Institute, Department of Physics and Astronomy, 251B Clippinger Lab, Ohio University, Athens, OH 45701, USA \\ ${ }^{8}$ Carnegie Observatories, 813 Santa Barbara Street, Pasadena, CA 91101, USA \\ ${ }^{9}$ Center for Cosmology and Particle Physics, New York University, 4 Washington Place, New York, NY 10003, USA \\ ${ }^{10}$ Laboratoire AIM (CEA/IRFU—CNRS/INSU—Université Paris Diderot), CEA DSM/IRFU/DAp, F-91191 Gif-sur-Yvette, France \\ ${ }^{11}$ Department of Physics \& Astronomy, Purdue University, West Lafayette, IN 47907, USA \\ ${ }^{12}$ National Science Foundation, 2415 Eisenhower Avenue, Alexandria, VA 22314, USA \\ Received 2017 November 8; revised 2018 February 2; accepted 2018 February 26; published 2018 March 26
}

\begin{abstract}
The energy source powering the extreme optical luminosity of hydrogen-stripped superluminous supernovae (SLSNe-I) is not known, but recent studies have highlighted the case for a central engine. Radio and/or X-ray observations are best placed to track the fastest ejecta and probe the presence of outflows from a central engine. We compile all the published radio observations of SLSNe-I to date and present three new observations of two new SLSNe-I. None were detected. Through modeling the radio emission, we constrain the subparsec environments and possible outflows in SLSNe-I. In this sample, we rule out on-axis collimated relativistic jets of the kind detected in gamma-ray bursts (GRBs). We constrain off-axis jets with opening angles of $5^{\circ}\left(30^{\circ}\right.$ ) to energies of $E_{\mathrm{k}}<4 \times 10^{50} \mathrm{erg}\left(E_{\mathrm{k}}<10^{50} \mathrm{erg}\right)$ in environments shaped by progenitors with mass-loss rates of $\dot{M}<10^{-4} M_{\odot} \mathrm{yr}^{-1}\left(\dot{M}<10^{-5} M_{\odot} \mathrm{yr}^{-1}\right)$ for all off-axis angles, assuming fiducial values $\epsilon_{e}=0.1$ and $\epsilon_{B}=0.01$. The deepest limits rule out emission of the kind seen in faint uncollimated GRBs (with the exception of GRB 060218) and from relativistic SNe. Finally, for the closest SLSN-I, SN 2017egm, we constrain the energy of an uncollimated nonrelativistic outflow like those observed in normal SNe to $E_{\mathrm{k}} \lesssim 10^{48} \mathrm{erg}$.
\end{abstract}

Key words: stars: jets - supernovae: general

\section{Introduction}

Superluminous supernovae (SLSNe) are a distinct class of supernovae (SNe) that have UV-optical luminosities $L>7 \times 10^{43} \mathrm{erg} \mathrm{s}^{-1}$ (Chomiuk et al. 2011; Quimby et al. 2011). These stellar explosions are typically $\sim 10-100$ times more luminous than ordinary $\mathrm{SNe},{ }^{14}$ show comparatively bright UV emission at early times, and, in some cases, have decay rates that are incompatible with ${ }^{56} \mathrm{Ni}$ and ${ }^{56} \mathrm{Co}$ decay (Gal-Yam 2012; De Cia et al. 2017; Lunnan et al. 2018).

There are two main classes of SLSNe, namely, the hydrogen-rich systems (SLSNe-II) and the hydrogen-stripped systems (SLSNe-I). Some SLSNe-II show clear signatures of shock interaction with a dense medium in their optical spectra (in the form of narrow emission lines with width $<100 \mathrm{~km} \mathrm{~s}^{-1}$ ). For these systems, the large UV-optical luminosity can be explained through the interaction of the blast wave with dense material left behind by the stellar progenitor before collapse (e.g., Ofek et al. 2007; Smith \& McCray 2007; Chatzopoulos et al. 2011). The mechanism or mechanisms that power the

\footnotetext{
${ }^{13}$ Hubble Fellow.

${ }^{14}$ Note that Milisavljevic et al. (2013) and Lunnan et al. (2018) found SNe with spectroscopic similarities to SLSNe but at lower luminosities.
}

exceptional luminosities of SLSNe-I, however, are unknown (e.g., Gal-Yam 2012).

A number of models for the energy source of SLSNe-I have been proposed. Higher luminosities could be explained by the presence of larger quantities of radioactive material (with respect to ordinary $\mathrm{SNe}$ ) or a central engine. Large quantities of ${ }^{56} \mathrm{Ni}$ could be produced by a pair-instability SN (Woosley et al. 2007; Gal-Yam et al. 2009). A central engine in the form of the spindown of a magnetar (e.g., Kasen \& Bildsten 2010; Woosley 2010; Nicholl et al. 2013; Metzger et al. 2015) or fallback accretion onto the compact remnant (Dexter \& Kasen 2013) has been suggested. The source of the large luminosity could also be due to increased efficiency of the conversion of kinetic energy into radiation via shock interaction in a particularly dense circumstellar medium (e.g., Smith \& McCray 2007; Chevalier \& Irwin 2011; Ginzburg \& Balberg 2012).

The mechanism (or mechanisms) powering the luminosity of SLSNe-I are a topic of debate. A key problem with the interaction model is that no clear evidence for a dense surrounding medium (such as narrow spectral lines with $v \leqslant 100 \mathrm{~km} \mathrm{~s}^{-1}$ at early times) has been observed in SLSNeI. Roth et al. (2016), however, showed that under the right conditions, the narrow-line emission could be suppressed. $\mathrm{H} \alpha$ 
emission has been detected at late times in three SLSNe-I (Yan et al. 2015, 2017a): to power this emission and not produce narrow lines, a few solar masses of hydrogen-free material would need to have been ejected in the last $~$ year before stellar explosion (e.g., Chevalier \& Irwin 2011; Chatzopoulos \& Wheeler 2012; Ginzburg \& Balberg 2012; Moriya et al. 2013). There are, however, claims that interaction of the ejecta with the medium is necessary to fit the light curves of some SLSNe-I, regardless of whether interaction is the dominant contribution to the flux (e.g., Yan et al. 2015; Wang et al. 2016; Tolstov et al. 2017).

Pair-instability SNe (Barkat et al. 1967) could produce the required amounts of ${ }^{56} \mathrm{Ni}$ to power the optical luminosity, but to date, only two candidates are known (Gal-Yam et al. 2009; Terreran et al. 2017), and the classification is debated in the literature (e.g., Yoshida \& Umeda 2011; Nicholl et al. 2013). If the radioactive decay of ${ }^{56} \mathrm{Ni}$ is the sole energy source, then for some SLSNe-I, the necessary quantities cannot be reconciled with the inferred ejecta mass, bright UV emission, or decay rate of the light curves (e.g., Kasen et al. 2011; Dessart et al. 2012; Inserra et al. 2013; Nicholl et al. 2013; McCrum et al. 2014; see, however, Kozyreva et al. 2017). Pair-instability explosions cannot account for the entire class of SLSNe-I.

Recent studies are increasingly favoring the central engine model (e.g., Margalit et al. 2018; Nicholl et al. 2017c), as it has been shown to satisfactorily reproduce the optical light curves of SLSNe-I with a wide range of properties (e.g., Chatzopoulos et al. 2013; Inserra et al. 2013; Nicholl et al. 2014, 2017b; Metzger et al. 2015; Inserra et al. 2017). Magnetar central engines with initial spin periods in the range $1-5 \mathrm{~ms}$ and magnetic fields in the range $\approx 10^{13}-10^{14} \mathrm{G}$ are the best fit for the optical bolometric emission of several systems (e.g., Dessart et al. 2012; Inserra et al. 2013; Nicholl et al. 2013; Metzger et al. 2015; Lunnan et al. 2016; Yu \& Li 2017).

There is growing evidence of a link between jetted long gamma-ray burst (GRB) SNe and SLSNe-I in the form of observational similarities in their spectra and light curves (e.g., Greiner et al. 2015; Metzger et al. 2015; Kann et al. 2016; Nicholl et al. 2016a; Jerkstrand et al. 2017), their preference for metal-poor host galaxies (e.g., Lunnan et al. 2014; Chen et al. 2015, 2017a, 2017c; Perley et al. 2016; Izzo et al. 2018; but also see Bose et al. 2018; Chen et al. 2017b; Nicholl et al. 2017a), ${ }^{15}$ and the models for the central engine (e.g., Metzger et al. 2015; Margalit et al. 2018). SLSNe and GRB SNe have broader spectral features than normal H-stripped SNe indicative of large photospheric velocities (Liu et al. 2017). Additionally, the luminous UV emission in the SLSN-I Gaia16apd has been suggested to originate from a central engine (Nicholl et al. 2017b; see, however, Yan et al. 2017b). In the SLSN-I SCP06F6, luminous X-ray emission (outshining even GRBs at a similar post-explosion time by a large factor), if indeed associated with the transient, is likely powered by a central engine (Gänsicke et al. 2009; Levan et al. 2013; Metzger et al. 2015). The presence of a central engine may also provide an additional driving force for the stellar explosion (e.g., Liu et al. 2017; Soker \& Gilkis 2017).

A key manifestation of a central engine is an associated jet. The search for evidence of a jet is best conducted at radio and $\mathrm{X}$-ray wavelengths. Optical emission is of thermal origin and tracks the slowly moving material in the explosion $(v \leqslant$ a few

\footnotetext{
15 See Lunnan et al. (2014), Leloudas et al. (2015), Angus et al. (2016), and Schulze et al. (2018) for a comparison of host properties.
}

$10^{4} \mathrm{~km} \mathrm{~s}^{-1}$ ). In contrast, radio and $\mathrm{X}$-ray emission are of nonthermal origin and arise from the interaction of the explosion's fastest ejecta $(v \geqslant 0.1 c)$ with the local environment. As the radiative properties of the shock front are directly dependent on the circumstellar density, radio/X-ray observations also probe the mass-loss history of the progenitor star in the years prior to explosion, a phase in stellar evolution that is poorly understood (see Smith 2014 for a recent review).

In Margutti et al. (2017a), we used the sample of X-ray observations of SLSNe-I to constrain relativistic hydrodynamical jet models and determine constraints on the central engines and subparsec environments of SLSNe-I. This work showed that interaction with a dense circumstellar medium is not likely to play a key role in powering SLSNe-I, and that at least some SLSN-I progenitors are compact stars surrounded by a low-density environment. There was no compelling evidence for relativistic outflows, but the limits were not sensitive enough to probe jets that were pointed more than $30^{\circ}$ out of our line of sight. In one case (PTF12dam), the X-ray limits were sufficiently deep to rule out emission similar to subenergetic GRBs, suggesting a similarity to the relativistic SNe 2009bb and 2012ap (Soderberg et al. 2010b; Margutti et al. 2014; Chakraborti et al. 2015) if this SLSN-I was a jet-driven explosion.

In this paper, we expand on recent analysis of radio observations from the SLSN 2015bn (Nicholl et al. 2016a; Margalit et al. 2018) and compile all the radio-observed SLSNe-I, including the three new observations presented for the first time in this work, with the aim of placing stronger constraints on the properties of their subparsec environment and fastest ejecta (both in the form of a relativistic jet and an uncollimated outflow). These data span 26-318 days after the explosion (in the explosion rest frame and at $\mathrm{GHz}$ frequencies). We test for the presence of a central engine that would produce a GRB-like jet and model the on-axis and off-axis emission from a jet for a range of densities, microphysical shock parameters, kinetic energies, jet opening angles, and off-axis observer angles and compare these to observations. We also explore the radio properties of uncollimated outflows that would be consistent with our limits and derive constraints on the fastest ejecta and mass-loss history of SLSNe-I.

In Section 2, we describe the sample of radio-observed SLSNe-I. In Section 3, we present our new radio observations of SLSNe-I and provide details on the data reduction. The constraints on on-axis and off-axis jets are given in Section 4. Section 5 describes the constraints we derive for uncollimated outflows. Conclusions are drawn in Section 6.

Unless otherwise stated, all time intervals and frequencies are quoted in the explosion rest frame, and the error bars are $1 \sigma$. We assume a $\Lambda$ cold dark matter cosmology with $H_{0}=70 \mathrm{~km} \mathrm{~s}^{-1} \mathrm{Mpc}^{-1}(h=0.7), \Omega_{0}=0.3$, and $\Omega_{\Lambda}=0.7$.

\section{Sample}

Our sample consists of all H-stripped SLSNe with published radio observations as of 2017 August, comprising nine SLSNe-I. This includes seven systems with radio observations already published in the literature (PS1-10ky, PS1-10awh, PS1-12fo, iPTF15cyk, SN 2015bn, PTF09cnd, and SN 2017egm) and two systems (PS1-10bzj and Gaia16apd) for which we present the first radio observations. We also present the latest observations of SN 2017egm, updating the observations from Bose et al. (2018). A brief description of each SLSN is given in the 
Table 1

Properties of the Sample of SLSNe-I

\begin{tabular}{|c|c|c|c|c|c|c|}
\hline Name(s) & $\begin{array}{c}d_{L} \\
(\mathrm{Mpc})\end{array}$ & $\begin{array}{l}\text { Explosion Date } \\
\text { (MJD) }\end{array}$ & $\begin{array}{c}\text { Time After Expl. }{ }^{\mathrm{a}} \\
\text { (days) }\end{array}$ & $\begin{array}{l}\text { Freq. }^{a} \\
(\mathrm{GHz})\end{array}$ & $\begin{array}{l}\text { Specific Luminosity } \\
(\mathrm{erg} / \mathrm{s} / \mathrm{Hz})\end{array}$ & References \\
\hline \multirow[t]{6}{*}{ PTF09cnd } & \multirow[t]{6}{*}{$1306^{\mathrm{b}}$} & \multirow[t]{6}{*}{$55006^{\mathrm{c}}$} & 85 & 10.6 & $<1.5 \times 10^{29}$ & Chandra et al. (2009b) \\
\hline & & & 85 & 6.1 & $<1.6 \times 10^{29}$ & Chandra et al. (2009b) \\
\hline & & & 85 & 1.8 & $<9.4 \times 10^{31 d}$ & Chandra et al. (2009b) \\
\hline & & & 140 & 10.6 & $<1.1 \times 10^{29}$ & Chandra et al. (2010) \\
\hline & & & 142 & 6.1 & $<2.4 \times 10^{29}$ & Chandra et al. (2010) \\
\hline & & & 147 & 1.8 & $<9.7 \times 10^{29 \mathrm{~d}}$ & Chandra et al. (2010) \\
\hline PS1-10awh & 5865 & 55467 & 39 & 9.6 & $<9.7 \times 10^{29}$ & Chomiuk et al. (2011) \\
\hline PS1-10bzj & $3891^{\mathrm{e}}$ & $55523^{\mathrm{e}}$ & 48 & 8.2 & $<8.6 \times 10^{29}$ & This work \\
\hline PS1-10ky & 6265 & $55299^{\mathrm{f}}$ & 156 & 9.6 & $<1.2 \times 10^{30}$ & Chomiuk et al. (2011) \\
\hline SN 2012i ${ }^{g}$ & $825^{\mathrm{h}}$ & $55919^{i}$ & 44 & 6.9 & $<1.5 \times 10^{28}$ & Chomiuk et al. (2012a) \\
\hline \multirow[t]{2}{*}{ SN 2015bn ${ }^{\mathrm{j}}$} & \multirow[t]{2}{*}{528} & \multirow[t]{2}{*}{$57013^{\mathrm{k}}$} & 318 & 8.2 & $<2.2 \times 10^{28}$ & Nicholl et al. (2016b) \\
\hline & & & 318 & 24.5 & $<1.2 \times 10^{28 \mathrm{~d}}$ & Nicholl et al. (2016b) \\
\hline \multirow[t]{3}{*}{ iPTF15cyk } & \multirow[t]{3}{*}{$3101^{1}$} & \multirow[t]{3}{*}{$57249^{\mathrm{m}}$} & 61 & 8.3 & $<2.2 \times 10^{29}$ & Palliyaguru et al. (2016), Kasliwal et al. (2016) \\
\hline & & & 94 & 8.3 & $<1.7 \times 10^{29}$ & Palliyaguru et al. (2016), Kasliwal et al. (2016) \\
\hline & & & 124 & 8.3 & $<1.7 \times 10^{29}$ & Palliyaguru et al. (2016), Kasliwal et al. (2016) \\
\hline \multirow[t]{4}{*}{ Gaia16apd $^{\mathrm{n}}$} & \multirow[t]{4}{*}{$467^{\circ}$} & \multirow[t]{4}{*}{$57512^{\mathrm{p}}$} & 26 & 6.6 & $<4.7 \times 10^{27}$ & This work \\
\hline & & & 26 & 24.0 & $<1.1 \times 10^{28 \mathrm{~d}}$ & This work \\
\hline & & & 203 & 6.6 & $<3.6 \times 10^{27}$ & This work \\
\hline & & & 203 & 24.0 & $<7.6 \times 10^{27 \mathrm{~d}}$ & This work \\
\hline \multirow[t]{7}{*}{ SN 2017egm ${ }^{q}$} & \multirow[t]{7}{*}{$136^{\mathrm{r}}$} & \multirow[t]{7}{*}{$57887^{\mathrm{s}}$} & 34 & 16.0 & $<3.9 \times 10^{28 \mathrm{~d}, \mathrm{t}}$ & Bright et al. (2017) \\
\hline & & & 38 & 5.2 & $<1.3 \times 10^{27 \mathrm{~d}}$ & Bright et al. (2017) \\
\hline & & & 39 & 10.3 & $<5.7 \times 10^{26, \mathrm{u}}$ & Romero-Canizales et al. (2017), Bose et al. (2018) \\
\hline & & & 39 & 1.6 & $<2.1 \times 10^{27 \mathrm{~d}}$ & Romero-Canizales et al. (2017), Bose et al. (2018) \\
\hline & & & 46 & 34.0 & $<3.3 \times 10^{27 \mathrm{~d}}$ & This work; Coppejans et al. (2017) \\
\hline & & & 47 & 10.3 & $<6.4 \times 10^{26, \mathrm{u}}$ & Romero-Canizales et al. (2017), Bose et al. (2018) \\
\hline & & & 74 & 34.0 & $<7.4 \times 10^{26 \mathrm{~d}}$ & This work \\
\hline
\end{tabular}

Notes. $3 \sigma$ upper limits are given on the luminosity.

${ }^{\mathrm{a}}$ Explosion rest frame.

${ }^{\mathrm{b}}$ Neill et al. (2011).

${ }^{\mathrm{c}}$ From Quimby et al. (2011), the peak time is MJD 55069.145 and assuming a rest-frame rise time of 50 days.

${ }^{\mathrm{d}}$ Not used in the modeling of emission from off-axis jets (Section 4), because the explosion rest-frame frequency is significantly different from $\sim 8 \mathrm{GHz}$.

e The peak was at MJD 55563.65+-2 (Lunnan et al. 2013), and as this was a fast-rising SN (R. Lunnan et al. 2018, in preparation), we assume a rest-frame rise time of 25 days.

${ }^{\mathrm{f}}$ Calculated based on the peak time from Chomiuk et al. (2011) and assuming a 50-day rise time in the explosion rest frame.

g Aliases: PS1-12fo and CSS120121:094613+195028.

${ }^{\text {h }}$ Smartt et al. (2012), Inserra et al. (2013).

${ }^{\mathrm{i}}$ Inserra et al. (2013).

j Aliases: PS15ae, CSS141223:113342+004332, and MLS150211:113342+004333.

${ }^{\mathrm{k}}$ The SN reached $r$-band maximum light on MJD 57102, and the inferred rise time in the explosion rest frame is $\sim 80$ days (Nicholl et al. $2016 \mathrm{~b}$ ).

${ }^{1}$ Kasliwal et al. (2016).

${ }^{\mathrm{m}}$ We estimated the peak time at 57293.5 MJD based on a comparison to LSQ12dlf (A. Corsi 2018, private communication) and assumed a rise time of 50 days in the explosion rest frame.

${ }^{\mathrm{n}}$ Alias: SN 2016eay.

${ }^{\circ}$ Nicholl et al. (2017b).

p Time of maximum light was MJD 57541 (Yan et al. 2015), and the rise time in the rest frame was 29 days (Nicholl et al. 2017b).

${ }^{\mathrm{q}}$ Alias: Gaia17biu.

${ }^{\mathrm{r}}$ Romero-Canizales et al. (2017).

${ }^{s}$ Nicholl et al. (2017a).

${ }^{\mathrm{t}}$ As the contribution of the galaxy (NGC 3191) to the detected flux density is unknown, we take it as an upper limit on the SN.

u These observations are presented in Bose et al. (2018) and Romero-Canizales et al. (2017). Individual upper limits for the two observations are from private communications with Subhash Bose and Cristina Romero-Canizales.

Appendix. Table 1 gives the radio observations and relevant references for all of these systems.

The exact date of explosion is not known for every object in this sample. In a number of cases, the times of the observations (in number of days since the explosion) were derived from the time of peak luminosity and an estimated rise time. Given the spread in rise times for SLSNe-I (see Nicholl et al. 2017c), the uncertainty on these derived observation times is less than 20 days. We tested the impact of this uncertainty on our conclusions in the following analysis by increasing and then decreasing the assumed rise times of all objects in our sample by 20 days. The differences in the constraints that we derive are marginal and do not affect our conclusions.

\section{Observations}

Our observations of SN 2017egm (NRAO observing code VLA/17A-466; PI: R. Margutti), Gaia16apd (VLA/16A-476; 
Table 2

Details of Observing Runs

\begin{tabular}{|c|c|c|c|c|c|c|c|c|}
\hline Name(s) & $\begin{array}{l}\text { Start Obs. } \\
\text { (MJD) }\end{array}$ & $\begin{array}{l}\text { Total Obs. }{ }^{\text {a }} \\
\text { Time (s) }\end{array}$ & $\begin{array}{l}\text { Frequency } \\
\quad(\mathrm{GHz})\end{array}$ & $\begin{array}{l}\text { Bandwidth } \\
\text { (GHz) }\end{array}$ & $\begin{array}{l}\text { VLA Array } \\
\text { Config. }\end{array}$ & $\begin{array}{c}\text { Beam Size } \\
\text { FWHM (arcsec) }\end{array}$ & $\begin{array}{l}\text { Beam Angle } \\
\text { (deg) }\end{array}$ & $\begin{array}{c}\text { Flux Density } \\
(\mu \mathrm{Jy})\end{array}$ \\
\hline$\overline{\text { PS1-10bzj }}$ & 55603.08146 & 2483 & 4.96 & 0.256 & C-CnB & $2.88 \times 1.30$ & -167.8 & $<87$ \\
\hline \multirow[t]{3}{*}{ Gaia16apd ${ }^{\mathrm{b}}$} & 57541.32332 & 1730 & 5.9 & 2.048 & $\mathrm{~B}$ & $2.28 \times 1.07$ & 69.1 & $<20.4$ \\
\hline & 57736.49994 & 1730 & 5.9 & 2.048 & A & $0.35 \times 0.32$ & 41.9 & $<15.3$ \\
\hline & 57736.47778 & 1731 & 21.8 & 2.048 & A & $0.11 \times 0.09$ & 58.7 & $<32.1$ \\
\hline SN $2017 \mathrm{egm}^{\mathrm{c}}$ & 57933.96987 & 2052 & 33.0 & 8.192 & $\mathrm{C}$ & $0.76 \times 0.62$ & 16.6 & $<150^{\mathrm{d}, \mathrm{e}}$ \\
\hline
\end{tabular}

Notes. Upper limits are $3 \sigma$.

a Including intervening calibrator scans but excluding initial setup scans.

${ }^{b}$ Alias: SN 2016eay.

c Alias: Gaia17biu.

d This observation was reported in an Astronomer's Telegram (Coppejans et al. 2017).

${ }^{\mathrm{e}}$ This observation had high noise levels due to poor weather conditions.

PI: R. Margutti), and PS1-10bzj (VLA/AS1020; PI: A. Soderberg) were taken with the Karl G. Jansky Very Large Array (VLA; Perley et al. 2011). Table 2 shows the details of the observations. These data were calibrated using the integrated VLA pipeline in $\mathrm{CASA}^{16} \mathrm{v}$ 4.7.0. The observations were taken in standard phase-referencing mode, and the absolute flux density scale was set via observations of a standard flux density calibrator (3C286 for Gaia16apd and 3C147 for PS1-10bzj and SN 2017egm) using the coefficients of Perley \& Butler (2013), which are within CASA. We used Briggs weighting with a robust parameter of one to image. Two Taylor terms were used to model the frequency dependence of the larger bandwidth observations (SN $2017 \mathrm{egm}$ and Gaia16apd). None of the sources were detected. We quote upper limits as 3 times the noise level in the vicinity of the source as derived from the CASA Imfit task. Our results are summarized in Tables 1 and 2 and Figure 1.

\section{Constraints on Relativistic Jets}

\subsection{On-axis Relativistic Jets}

Figure 1 shows the $\sim 6-10 \mathrm{GHz}$ SLSN-I radio luminosity upper limits in reference to those from other classes of massive stellar explosions from H-stripped progenitors, including long GRBs (hereafter referred to just as GRBs), "normal" H-poor core-collapse SNe (Type Ibc; see Filippenko 1997), and relativistic SNe. On-axis jets in GRBs (both collimated and poorly collimated systems like subenergetic GRBs 980425 , 060218, and 100316D in Figure 1) produce luminous radio emission (e.g., Kulkarni et al. 1998; Soderberg et al. 2006b, 2010a; Chandra et al. 2009a).

The SLSN-I radio luminosity limits are significantly fainter than most cosmological GRBs detected in the radio, which typically show $L_{\nu} \geqslant 10^{29} \mathrm{erg} \mathrm{s}^{-1} \mathrm{~Hz}^{-1}$ (Figure 1). Our deepest luminosity limits acquired for SN 2017egm are deeper than the deepest limits for the sample of radio-observed GRBs in Chandra \& Frail (2012; see their Figure 6). SN 2017egm (and most of our sample of SLSNe-I) is significantly closer than cosmological GRBs (see Chandra \& Frail 2012). We restrict

\footnotetext{
${ }^{16}$ Common Astronomy Software Applications package (McMullin et al. 2007).
}

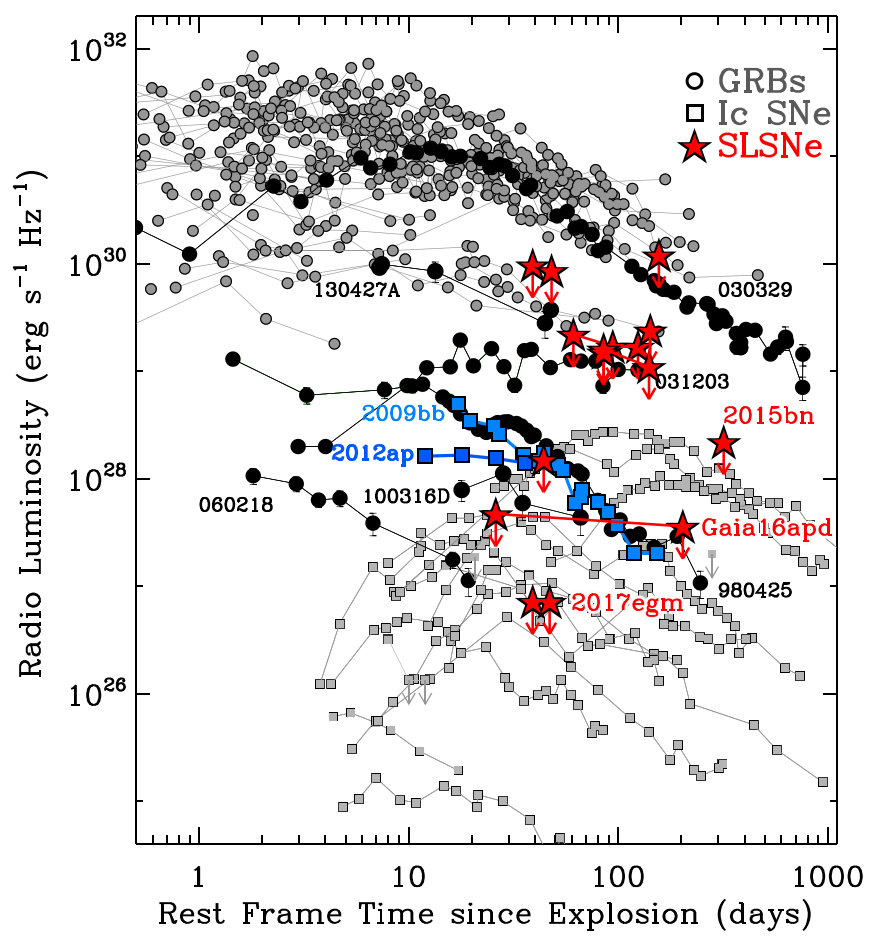

Figure 1. Specific radio luminosity at $\sim 8 \mathrm{GHz}$ (rest frame) for SLSNe-I (red stars) in the context of H-stripped core-collapse explosions (i.e., GRBs (circles) and normal Ic $\mathrm{SNe}$ (squares)). Black circles: GRBs at $z \leqslant 0.3$. Gray circles: GRBs at $z>0.3$. Gray squares: normal Ic SNe. Blue squares: relativistic Ic SNe. Connected symbols refer to observations of the same object. For display purposes, only the SLSNe-I directly referred to in the text are labeled. Deep radio observations of the closest SLSNe-I, like Gaia16apd and SN 2017egm, clearly rule out on-axis jets of the kind detected in GRBs and probe the parameter space of the weakest engine-driven $\mathrm{SNe}$ (like those associated with GRBs 980425 and 100316D). Notably, radio observations of Gaia16apd and SN 2017egm indicate that SLSNe-I can be significantly fainter than normal $\mathrm{H}$-stripped core-collapse $\mathrm{SNe}$ as well. References: Immler et al. (2002), Pooley \& Lewin (2004), Soria et al. (2004), Soderberg et al. (2005), Perna et al. (2008), Chandra et al. (2009b, 2010), Soderberg et al. (2010b), Corsi et al. (2011), Chomiuk et al. (2011), Chomiuk et al. (2012a), Chandra \& Frail (2012), Horesh et al. (2013), Margutti et al. (2013b, 2013a, 2014), Corsi et al. (2014), Nicholl et al. (2016b), Kasliwal et al. (2016), Palliyaguru et al. (2016), Bright et al. (2017), Bose et al. (2018), Coppejans et al. (2017), Romero-Canizales et al. (2017).

the comparison of our SLSN radio limits to the sample of GRBs in the local universe $(z<0.3)$, which are more representative of the true demographics (at higher redshifts $z>0.3$, we are sensitive only to the high-energy tail of the 
GRB distribution). ${ }^{17}$ At $z<0.3$, we are consequently sensitive to the entire demographics of long GRBs. For Gaia16apd and SN 2017egm, our limits rule out radio emission of the kind detected from GRBs in the local universe (black points in Figure 1), with the exception of the faint GRB 060218 (for which there is no evidence for collimation of the fastest ejecta).

Notably, for Gaia16apd and SN 2017egm, we can rule out emission of the kind detected from the low-luminosity GRB 980425 associated with SN 1998bw (for which there is also no evidence for collimation of the fastest ejecta). This is of particular relevance, as Nicholl et al. (2016b) found clear similarities in the nebular spectra of the SLSN 2015bn and the GRB SN 1998bw, which suggests a similar core structure of their stellar progenitors at the time of collapse and possibly also a similar explosion central engine. Radio observations show that this similarity does not extend to the properties of the fastest ejecta of Gaia16apd and SN 2017egm. (We note that for SLSN 2015bn, radio observations were acquired at a much later epoch and do not constrain GRB 980425-like radio emission, as shown in Figure 1; Nicholl et al. 2016a). If the deepest limits (SN 2017egm and Gaia16apd) are excluded, the rest of the sample still rules out emission of the kind seen in some of the low-luminosity GRBs.

Radio observations of SN 2017egm were acquired at later times than those of the faint GRBs, leaving the possibility of a GRB 060218-like outflow in SLSNe-I still open. To determine the presence of GRB 060218-like emission in SLSNe-I, radio observations at $\lesssim 10$ days after the explosion are necessary. This can be seen by considering GRB 060218 in Figure 1: in GRB 060218 , the radio luminosity declined by approximately an order of magnitude in the first $\sim 30$ days after the explosion, which is the earliest phase for which we have SLSN-I radio observations.

We conclude that this sample of SLSNe-I is not consistent with having on-axis jets of the kind detected in GRBs. The deepest SLSN-I limits also rule out emission from weak, poorly collimated GRBs, with the notable exception of the fast-fading GRB 060218.

\subsection{Off-axis Relativistic Jets}

\subsubsection{Simulation Setup}

To constrain the presence of off-axis relativistic outflows in SLSNe-I, we generated a grid of model light curves for off-axis GRB jets using high-resolution, two-dimensional relativistic hydrodynamical jet simulations. For this, we used the broadband afterglow numerical code Boxfit v2 (van Eerten et al. 2012), which models the off-axis, frequency-dependent emission as the jet slows and the radiation becomes less beamed. We then compared the collective $\sim 8 \mathrm{GHz}$ (central frequencies in the range $6.1-10.6 \mathrm{GHz}$, as indicated in Table 1) radio upper limits in our sample to each light curve to determine if the observations rule out that particular set of parameters. These frequencies provide the most stringent constraints on the jet parameters, as they include the deepest limits, were taken at the earliest and latest times, and have the densest time coverage. To do this, we made the necessary assumption that every SLSN-I in our sample is powered by the same mechanism (and jet/environment properties), i.e., a given

\footnotetext{
17 Since the VLA upgrade, more sensitive observations of GRBs at $z<0.3$ have consistently yielded detections (Zauderer et al. 2012; van der Horst 2013; Horesh et al. 2015; Kamble 2015; Laskar et al. 2016).
}

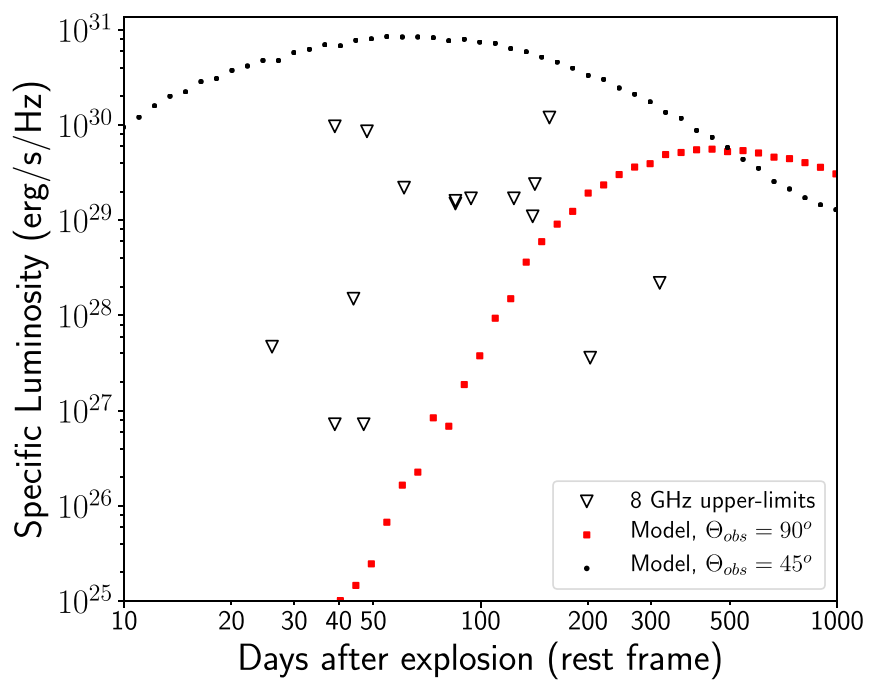

Figure 2. Example illustrating how the collective SLSN-I $\sim 8 \mathrm{GHz}$ limits and the model jet light curves are used to test a set of parameters. Two models for off-axis jets are shown for an ISM profile CSM (constant density, $\rho_{\mathrm{CSM}}$ ), with $\theta_{\mathrm{j}}=30^{\circ}, E_{\mathrm{k}, \text { iso }}=10^{53} \mathrm{erg}, n_{\mathrm{CSM}}=10 \mathrm{~cm}^{-3}, \epsilon_{e}=0.1$, and $\epsilon_{B}=0.01$. The red squares and black dots show the emission for the jet positioned at angles of $\theta_{\mathrm{obs}}=90^{\circ}$ and $\theta_{\mathrm{obs}}=45^{\circ}$, respectively. The radio limits rule out this set of parameters for both models, as they are lower than the predicted specific luminosities for either angle. The radio emission from a jet at a larger off-axis angle will peak later than it would at smaller angles, as the radiation is initially beamed away from the observer and will take longer to spread into the line of sight. Late-time observations are consequently necessary to constrain off-axis jets. For this set of parameters, the latest two observations (at 203 and 318 days for Gaia16apd and SN 2015bn, respectively) are more constraining for the $\theta_{\text {obs }}=90^{\circ}$ jet than the deepest radio limits, as the emission peaks later on.

set of parameters is ruled out if they are ruled out for at least one SLSN-I. The radio light curves are not sufficiently wellsampled to do this analysis individually. An illustration of this process is given in Figure 2.

The modeled radio light curves depend on the following input parameters: (1) the isotropic equivalent kinetic energy $\mathrm{E}_{\mathrm{k} \text {,iso }}$ of the outflow; (2) the density of the medium, where either an interstellar medium (ISM)-like medium ( $n_{\mathrm{CSM}}$ constant) or a wind-like medium $\left(\rho_{\mathrm{CSM}}=\dot{M} /\left(4 \pi R^{2} v_{\mathrm{w}}\right)\right)$ produced by a constant progenitor mass-loss rate $\dot{M}$ can be chosen; (3) the microphysical shock parameters $\epsilon_{B}$ and $\epsilon_{e}$, which are the postshock energy fraction in the magnetic field and electrons, respectively (see Sironi et al. 2015 for more details); (4) the jet opening angle $\theta_{\mathrm{j}}$; and (5) the observer angle with respect to the jet axis $\theta_{\text {obs }}$ (hereafter referred to as the observer angle). We fixed the power-law index of the shocked electron energy distribution to $p=2.5$, as it typically varies in the range 2-3 from GRB afterglow modeling (e.g., Curran et al. 2010 and Wang et al. 2015). Unless otherwise specified, we will report mass-loss rates $\dot{M}$ for an assumed wind velocity of $v_{\mathrm{w}}=1000 \mathrm{~km} \mathrm{~s}^{-1}$, which is representative of compact massive stars like Wolf-Rayet stars.

We explore two physical scenarios for the interstellar medium, namely, ISM-like $\left(10^{-3} \mathrm{~cm}^{-3} \leqslant n_{\mathrm{CSM}} \leqslant 10^{2} \mathrm{~cm}^{-3}\right)$ and wind-like $\left(10^{-8} M_{\odot} \mathrm{yr}^{-1} \leqslant \dot{M} \leqslant 10^{-3} M_{\odot} \mathrm{yr}^{-1}\right)$, for two jet collimation angles $\left(\theta_{\mathrm{j}}=5^{\circ}\right.$ and $\left.30^{\circ}\right)$, three observer angles $\left(\theta_{\mathrm{obs}}=30^{\circ}, 60^{\circ}\right.$, and $\left.90^{\circ}\right)$ and isotropic kinetic energies in the range $10^{50} \mathrm{erg} \leqslant E_{\mathrm{k} \text {,iso }} \leqslant 10^{55} \mathrm{erg}$. These values are representative of the parameters that are derived from accurate modeling of the broadband afterglows of GRBs (e.g., Schulze 


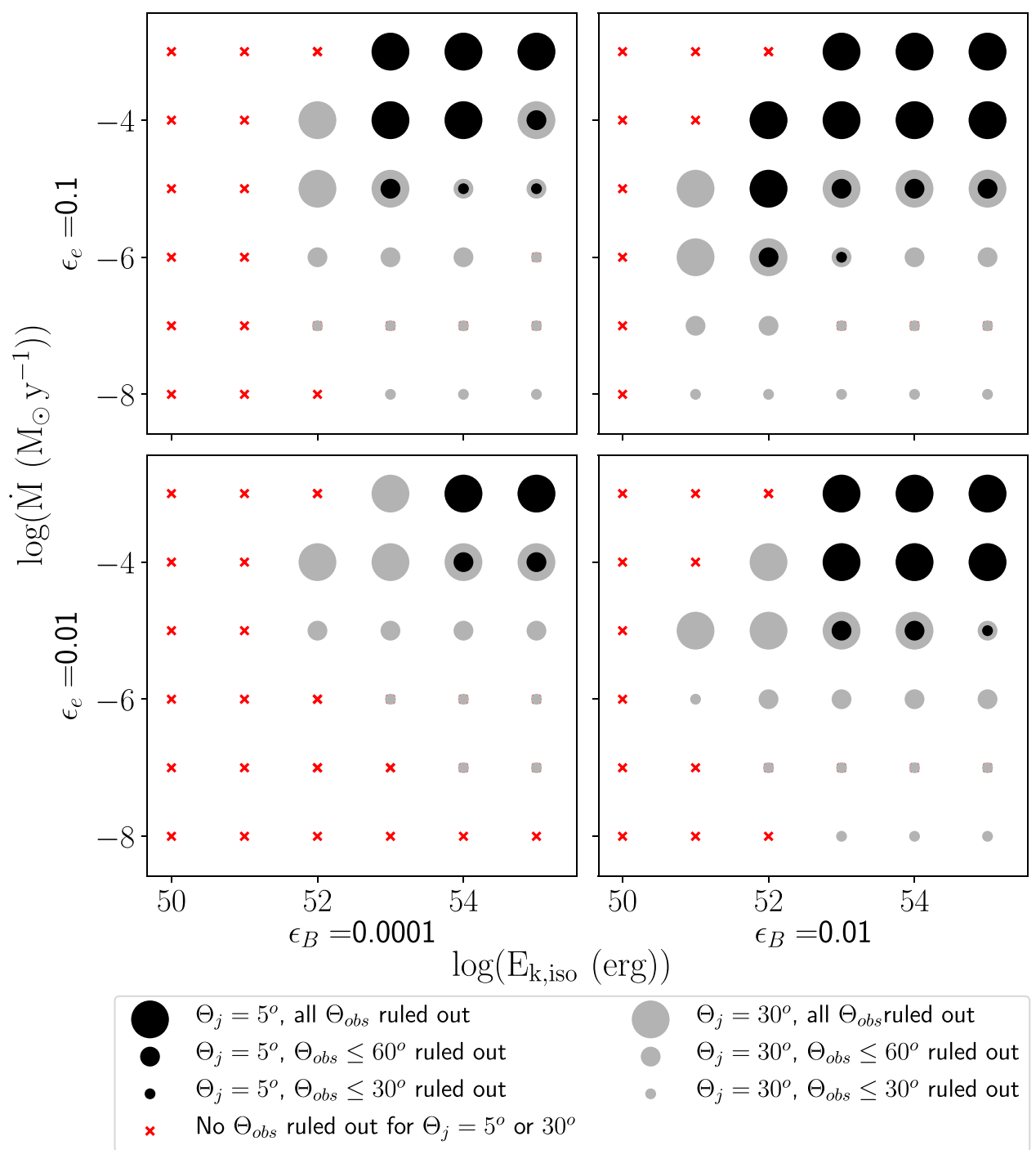

Figure 3. Constraints on jetted outflows in the sample of radio-observed SLSNe-I, assuming the progenitor produced a wind density profile $\left(\rho \propto r^{-2}\right)$ in the surrounding medium. The symbol colors represent jet opening angles of $\theta_{\mathrm{j}}=5^{\circ}$ (black) and $\theta_{\mathrm{j}}=30^{\circ}$ (gray). Symbol sizes indicate the observer angle $\left(\theta_{\mathrm{obs}}\right)$ for which we can rule out the corresponding jet, with larger symbols corresponding to larger $\theta_{\text {obs }}$. Red crosses indicate that the parameters could not be ruled out. The top (bottom) panels are $\epsilon_{e}=0.1\left(\epsilon_{e}=0.01\right)$, and the left (right) panels are $\epsilon_{B}=0.0001\left(\epsilon_{B}=0.01\right)$. Note: In the top left panel, highly collimated jets $\left(\theta_{\mathrm{j}}=5^{\circ}\right)$ with $E_{\mathrm{k}, \text { iso }} \geqslant 10^{53} \mathrm{erg}$ and progenitor mass-loss rates of $\dot{M} \geqslant 10^{-4} M_{\odot} \mathrm{yr}^{-1}$ are ruled out for all observer angles. The "outlier" at $E_{\mathrm{k} \text {,iso }}=10^{55} \mathrm{erg}$ was a sampling effect, where the upper limit was negligibly more luminous than the model at $\theta_{\mathrm{obs}}=90^{\circ}$.

et al. 2011; Laskar et al. 2013; Perley et al. 2014; Laskar et al. 2016).

In Figures 3 and 4, we present the results from the entire set of simulations for the range of $\epsilon_{e}$ and $\epsilon_{B}$ typically used in the literature. Relativistic shock simulations show $\epsilon_{e}=0.1$ (e.g., Sironi et al. 2015), and $\epsilon_{B}$ is less constrained than $\epsilon_{e}$. The distribution for $\epsilon_{B}$ derived from GRB afterglow modeling is centered on 0.01 and typically spans $10^{-4}$ to 0.1 , with a few claims for smaller values down to $\approx 10^{-7}$ (e.g., Santana et al. 2014). In the text, we discuss the results for the fiducial parameters $\epsilon_{\mathrm{e}}=0.1$ and $\epsilon_{B}=0.01$ but show the results for other typical values of the microphysical shock parameters in the figures.

At radio frequencies, the afterglow radiation (i.e., radiation arising from the jet interaction with the medium) consists of synchrotron emission. Both synchrotron emission and synchrotron self-absorption (SSA) are accounted for in the afterglow models. Free-free absorption is not significant for the circumstellar medium (CSM) densities and blast-wave velocities that we consider here. Following Weiler et al. (1986), and considering a wind medium with the highest mass-loss rates investigated here (i.e., $\dot{M}=10^{-3} M_{\odot} \mathrm{yr}^{-1}$ ), we find the freefree optical depth $\tau_{\mathrm{ff}}<0.04$ for frequencies greater than $5 \mathrm{GHz}$ at time $t>26$ days. The SLSN-I 2017egm was observed at $1.6 \mathrm{GHz}$ at $\sim 39$ days since explosion (Table 1). In this case, we estimate a $<15 \%$ flux reduction due to free-free absorption for the largest densities considered in this study, with no impact on our major conclusions. For the ISM-like densities considered below, free-free absorption is always negligible.

We consider the radio limits from the entire sample of SLSNe-I in this analysis. Note that the constraints that we derive are not driven solely by one SLSN-I. Although the limits for SN 2017egm are significantly deeper than for the rest of the sample, we only have early-time coverage for this system. As late-time observations are more constraining for off-axis jets, the other systems in our sample still provide meaningful constraints for off-axis jets (see Figure 2). SN 2017 egm exploded in 2017 June, so our limits only extend to 


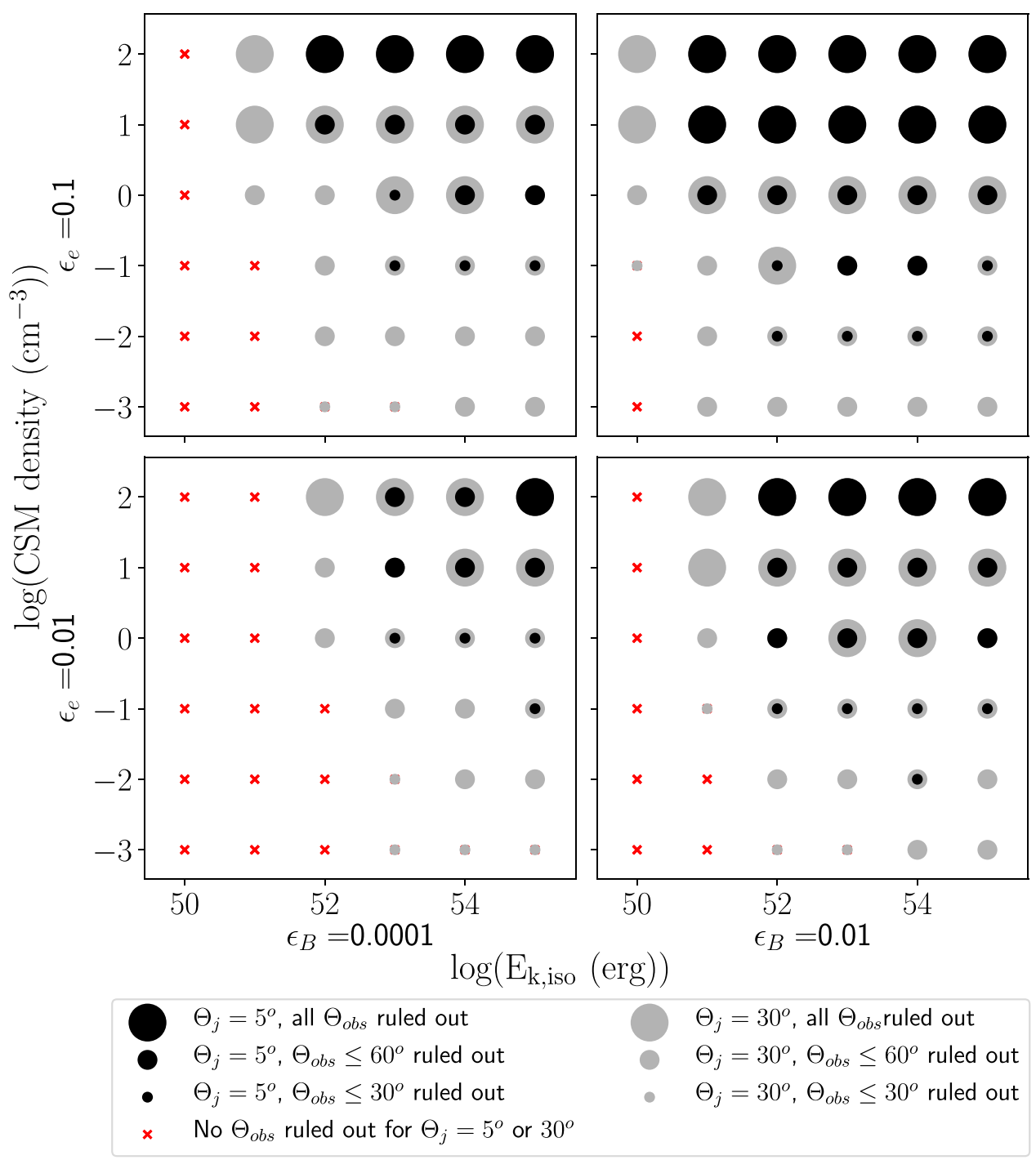

Figure 4. Constraints on jetted outflows in the sample of radio-observed SLSNe-I for a constant density profile in the surrounding medium. See the caption of Figure 3 for a full description of the symbols. Note: In the top right panel, highly collimated jets $\left(\theta_{\mathrm{j}}=5^{\circ}\right)$ with $E_{\mathrm{k}, \text { iso }} \geqslant 10^{51}$ erg in environments with $n_{\mathrm{CSM}}=10 \mathrm{~cm}{ }^{-3}$ are ruled out for all observer angles.

47 days (considering only the $\sim 8 \mathrm{GHz}$ observations). Radio observations of this object at later times will place the strongest constraints on off-axis jets in SLSNe-I to date. We will consequently continue radio monitoring of SN 2017egm.

\subsubsection{Results: $E_{k \text {,iso }}$ and $\dot{M}$ Phase Space}

Figures 3 and 4 show the constraints that the upper limits on the radio luminosity of the sample of SLSNe-I place on off-axis jets expanding into a wind profile medium and an ISM profile medium, respectively. First, consider a wind profile medium and jets that are off-axis and highly collimated $\left(\theta_{\mathrm{j}}=5^{\circ}\right.$, like those detected in GRBs). For $\epsilon_{\mathrm{e}}=0.1$ and $\epsilon_{B}=0.01$ (top right panel), these off-axis GRB-like jets are ruled out regardless of the observer angle for $\dot{M} \gtrsim$ $10^{-4} M_{\odot} \mathrm{yr}^{-1}$ and $E_{\mathrm{k} \text {,iso }} \gtrsim 10^{53} \mathrm{erg}$ (this is within the energy range of the observed GRB population). Mass-loss rates such as these are typically found in the winds of extreme red supergiants (e.g., Smith et al. 2009; Smith 2014) and luminous blue variables (Groh 2014; Smith 2014). To put this in context, Figure 5 shows the mass-loss rates and equivalent densities at $10^{16} \mathrm{~cm}$ (assuming a $1000 \mathrm{~km} \mathrm{~s}^{-1}$ progenitor wind speed) for other H-poor stellar explosions. Specifically, mass-loss rates of this order $\left(\dot{M} \gtrsim 10^{-4} M_{\odot} \mathrm{yr}^{-1}\right)$ have been inferred for some SNe Ibc (Figure 5 and references therein), as well as for $\mathrm{SNe} \mathrm{IIb}^{18}$ with yellow supergiant progenitors (e.g., SN 2013 $\mathrm{df}^{19}$; Kamble et al. 2016). The precluded

phase space for highly collimated, off-axis jets with $\dot{M} \gtrsim$ $10^{-4} M_{\odot} \mathrm{yr}^{-1}$ and $E_{\mathrm{k} \text {,iso }} \gtrsim 10^{53}$ is indicated in Figure 5. GRBlike jets are not ruled out for the lower-density environments inferred for some GRBs.

If instead we consider off-axis jets that are less collimated $\left(\theta_{\mathrm{j}}=30^{\circ}\right)$ than cosmological GRBs, we can probe to deeper limits, as the jet is less collimated to start with and more kinetic energy is coupled to it (with respect to a more collimated jet with the same $E_{\mathrm{k}, \text { iso }}$ ). In this case, regardless of the observing angle, we can rule out scenarios where $\dot{M} \gtrsim 10^{-5} M_{\odot} \mathrm{yr}^{-1}$ and $E_{\mathrm{k}, \text { iso }} \gtrsim 10^{53} \mathrm{erg}\left(E_{\mathrm{k}}<10^{50} \mathrm{erg}\right.$ ), as shown in Figure 3 (for

\footnotetext{
18 Type IIb SNe are a class that originally shows $\mathrm{H}$ lines but transitions to Type Ib-like (no $\mathrm{H}$ lines) over time.

19 Note that we assume a $1000 \mathrm{~km} \mathrm{~s}^{-1}$ wind here and have adjusted the massloss rate in Kamble et al. (2016) accordingly.
} 


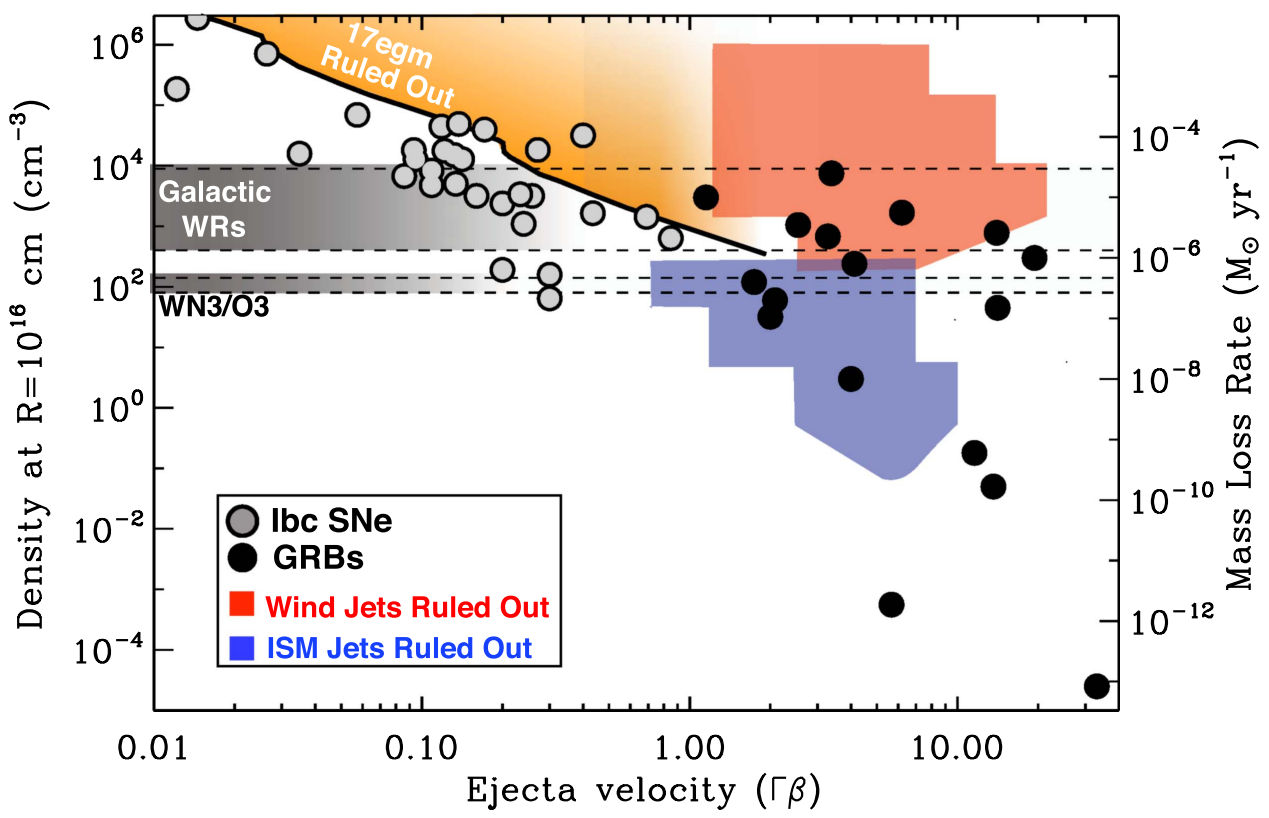

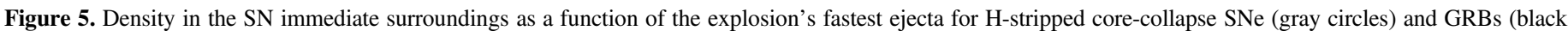

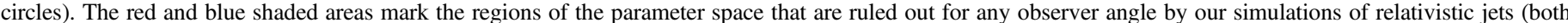

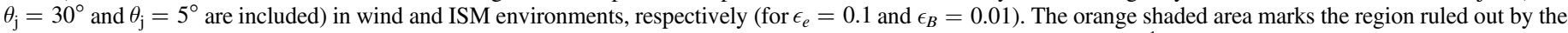

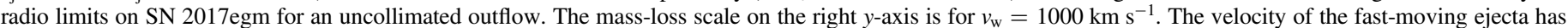

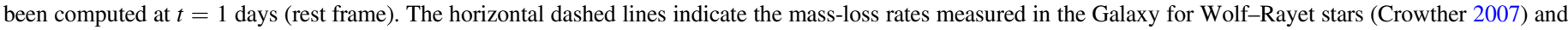

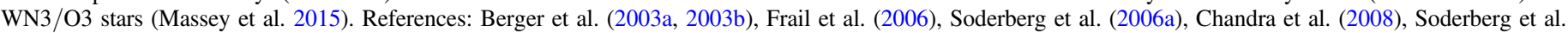

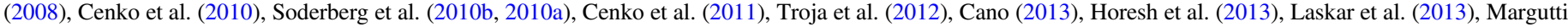
et al. (2013a, 2014), Perley et al. (2014), Walker et al. (2014), Chakraborti et al. (2015), Milisavljevic et al. (2015).

$\epsilon_{e}=0.1$ and $\left.\epsilon_{B}=0.01\right)$. This parameter space is illustrated in Figure 5. A significant fraction of the galactic Wolf-Rayet population (Vink \& de Koter 2005; Crowther 2007) and luminous blue variables (e.g., Vink \& de Koter 2002; Smith et al. 2004), as well as the most luminous O-type stars (e.g., de Jager et al. 1988; van Loon et al. 2005), show mass-loss rates in this range. Off-axis jets of this kind with $E_{\mathrm{k}, \text { iso }} \gtrsim 10^{53} \mathrm{erg}$ would also be precluded in the most dense environments inferred for GRBs and for most of the observed population of hydrogen-stripped SNe (assuming a $1000 \mathrm{~km} \mathrm{~s}^{-1}$ wind).

In Section 4.1, we discussed how this sample of SLSNe-I ruled out on-axis jets of the kind seen in low-luminosity (lesscollimated) GRBs, with the exception of GRB 060218. Now consider less-collimated jets $\left(\theta_{\mathrm{j}}=30^{\circ}\right)$ that are aligned only slightly off-axis-within $30^{\circ}$ of our line of sight. Jets of this kind are ruled out down to clean environments of $\dot{M} \gtrsim 10^{-8} M_{\odot} \mathrm{yr}^{-1}$, where $E_{\mathrm{k}, \text { iso }} \gtrsim 10^{51} \mathrm{erg}$ (Figure 3 , for $\epsilon_{e}=0.1$ and $\left.\epsilon_{B}=0.01\right)$. Assuming a progenitor wind speed of $1000 \mathrm{~km} \mathrm{~s}^{-1}$, this parameter space precludes the environments of all of the detected SNe Ibc and most of the GRBs detected to date (see Figure 5).

For comparison, Figure 4 gives the equivalent constraints for a constant density environment (modeling of GRB afterglows sometimes indicates a better fit to ISM environments; e.g., Laskar et al. 2014). For $\epsilon_{e}=0.1$ and $\epsilon_{B}=0.01$ (top right panel), a collimated jet with $\theta_{\mathrm{j}}=5^{\circ}$ is ruled out regardless of the observer angle for $n_{\mathrm{CSM}} \gtrsim 10 \mathrm{~cm}^{-3}$ and $E_{\mathrm{k} \text {,iso }} \gtrsim 10^{51} \mathrm{erg}$. A jet with $\theta_{\mathrm{j}}=30^{\circ}$ is ruled out for $n_{\mathrm{CSM}} \gtrsim 1 \mathrm{~cm}^{-3}$ and $E_{\mathrm{k}, \text { iso }} \gtrsim 10^{51} \mathrm{erg}$. Deeper constraints are obtained for jets with their axes aligned within $30^{\circ}$ or $60^{\circ}$ of our line of sight. Specifically, the jets with $\theta_{\mathrm{j}}=5^{\circ}$ and observer angles of $\leqslant 30^{\circ}$ are excluded down to $n_{\mathrm{CSM}} \gtrsim 10^{-3} \mathrm{~cm}^{-3}$.

\subsubsection{Results: $E_{k}$ and $\Gamma \beta$ Phase Space}

Engine-driven explosions (i.e., GRBs, subenergetic GRBs, and relativistic $\mathrm{SNe}$ ) are clearly distinguished from normal spherical core-collapse SNe by a flatter kinetic energy profile of their ejecta (e.g., Soderberg et al. 2006a). For an engine-driven explosion, a larger fraction of the kinetic energy is contained in the fast-moving ejecta than in the slow-moving ejecta, in contrast to a hydrodynamical explosion. This is illustrated in Figure 6, where we plot the kinetic energy in the slow- and fastmoving ejecta (joined by a dashed line to guide the eye) for the $\mathrm{H}$-poor explosions where these properties have been measured. For a pure hydrodynamical explosion, we would expect a profile of $E_{\mathrm{k}} \sim(\Gamma \beta)^{-5.2}$, while GRBs have significantly flatter profiles (Tan et al. 2001). A flat energy profile for the SLSNe-I would suggest an engine-driven explosion.

We have excluded a region of the $E_{\mathrm{k}}$-versus- $\Gamma \beta$ phase space in Figure 6 for this sample of SLSNe-I based on the limits from our simulations. Specifically, for a collimated $\left(\theta_{\mathrm{j}}=30^{\circ}\right)$ jet (ruled out at all observer angles), we deduce limits based on the excluded combinations of mass-loss rate and isotropic kinetic energy (Figures 3 and 4) as follows. Applying the standard formulation of the fireball dynamics with expansion in a windlike and ISM-like environment (e.g., Chevalier \& Li 2000), the bulk Lorentz factor of the downstream fluid behind the shock front is

$$
\Gamma \sim 18.7\left(E_{\mathrm{k}, \text { iso }} / 10^{54} \mathrm{erg}\right)^{1 / 4}\left(A_{*} / 0.1\right)^{-1 / 4}(t / 1 \text { day })^{-1 / 4}
$$

for a wind profile medium and

$\Gamma \sim 10.1\left(E_{\mathrm{k}, \text { iso }} / 10^{54} \mathrm{erg}\right)^{1 / 8}\left(n_{\mathrm{CSM}} / 0.1 \mathrm{~cm}^{-3}\right)^{-1 / 8}(t / 1 \text { day })^{-3 / 8}$

for an ISM profile medium. Here $\Gamma_{s}=\sqrt{2} \Gamma$ is the shock Lorentz factor, $A_{*}$ is the wind parameter characterizing 


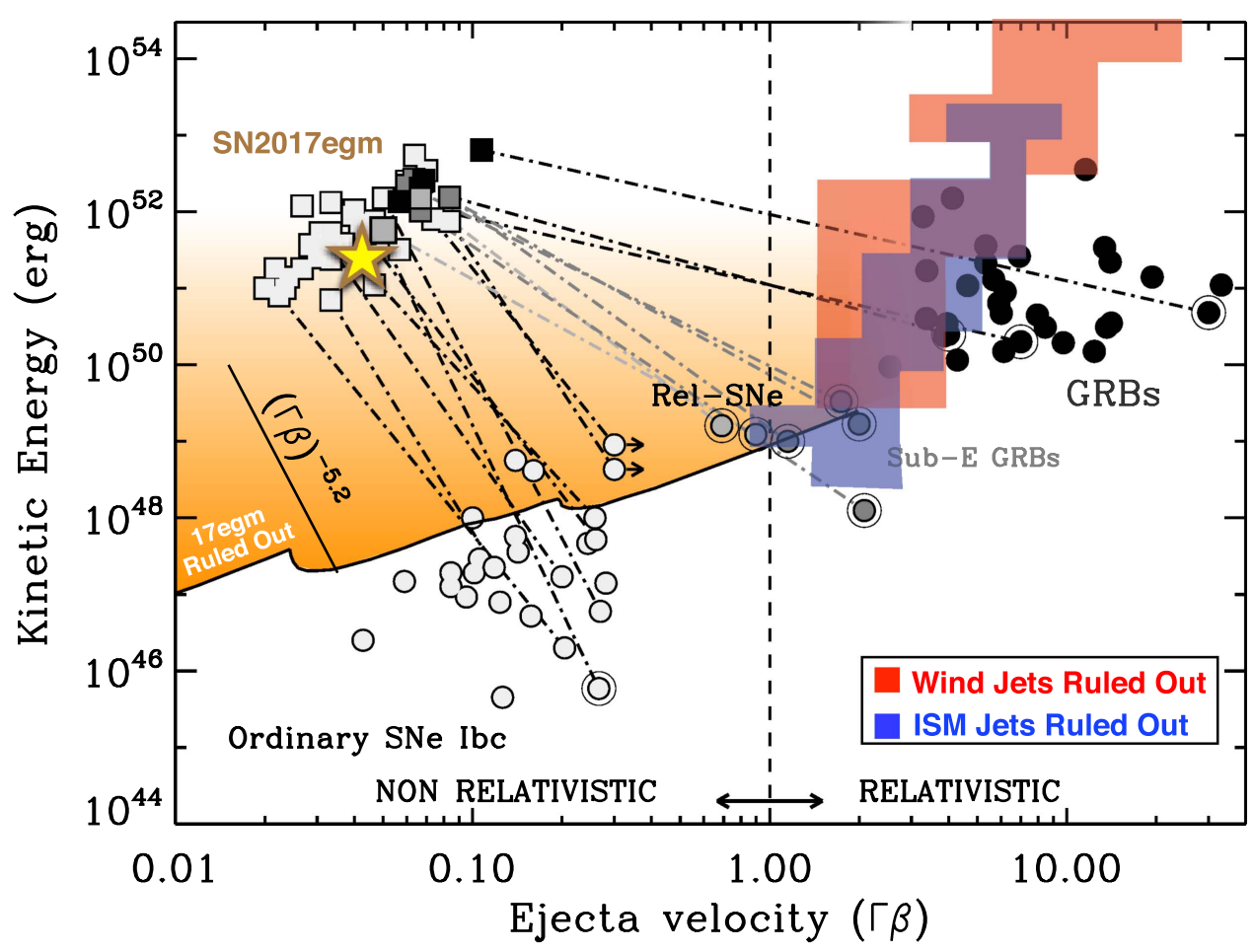

Figure 6. Kinetic energy profile of the ejecta of H-poor cosmic explosions, including ordinary Type Ibc SNe, relativistic SNe, GRBs, and subenergetic GRBs. The symbol colors indicate the class of object, namely, black for GRBs, gray for relativistic SNe, and white for ordinary SNe Ibc. Shaded areas mark the constraints on the properties of the SLSN-I fastest ejecta. Squares and circles are used for the slow-moving and fast-moving ejecta, respectively, as measured from optical (slow ejecta) and radio (fast ejecta) observations. An additional circle surrounding a point indicates that the object showed a broad-line optical spectrum. The velocity of the fastmoving ejecta has been computed at $t=1$ days (rest frame). The ejecta kinetic energy profile of a pure hydrodynamical explosion is also marked as a reference $\left(E_{k} \sim(\Gamma \beta)^{-5.2}\right.$; Tan et al. 2001). The blue and red areas identify the regions of the parameter space of the fast-moving ejecta that are ruled out based on our simulations of relativistic jets expanding in an ISM and wind-like environments, respectively (for $\epsilon_{e}=0.1$ and $\epsilon_{B}=0.01$ ). Only jet models that are ruled out for any observer angle are shown here. The orange shaded area identifies the region of the parameter space that is ruled out based on our simulations of radio emission from noncollimated outflows and the radio limits on SN 2017egm. The location of the slowly moving ejecta of SN 2017egm is shown with a star. References: Berger et al. (2003a, 2003b), Frail et al. (2006), Soderberg et al. (2006a), Chandra et al. (2008), Soderberg et al. (2008), Cenko et al. (2010), Soderberg et al. (2010b, 2010a), Cenko et al. (2011), Ben-Ami et al. (2012), Sanders et al. (2012), Troja et al. (2012), Cano (2013), Horesh et al. (2013), Laskar et al. (2013), Margutti et al. (2013a), Mazzali et al. (2013), Milisavljevic et al. (2013), Xu et al. (2013), Corsi et al. (2014), Guidorzi et al. (2014), Kamble et al. (2014), Margutti et al. (2014), Perley et al. (2014), Walker et al. (2014), Chakraborti et al. (2015), Milisavljevic et al. (2015), Bose et al. (2018), Nicholl et al. (2017a).

the density of the wind-generated CSM, and $A_{*}=1$ for $\dot{M}=10^{-5} M_{\odot} \mathrm{yr}^{-1}$ and $v_{\mathrm{w}}=1000 \mathrm{~km} \mathrm{~s}^{-1}$. In Figure 6, we plot the beaming-corrected kinetic energy $E_{\mathrm{k}}=E_{\mathrm{k} \text {,iso }}$ $\left(1-\cos \theta_{\mathrm{j}}\right)$ and estimate the specific momentum of the fastest ejecta at an arbitrary time of 1 day post-explosion (rest frame). The excluded phase spaces for a jet collimated to $\theta_{\mathrm{j}} \leqslant 30^{\circ}$ are shaded in red and blue in Figure 6 for a wind and ISM medium, respectively.

The excluded phase space does not constrain the slope of the kinetic energy profile to the extent where we can confirm or rule out the presence of a central engine in this sample of SLSNe-I. Based on our simulations, we ruled out off-axis collimated $\left(\theta_{\mathrm{j}}=30^{\circ}\right)$ jets at $\dot{M} \gtrsim 10^{-5} M_{\odot} \mathrm{yr}^{-1}$ and $E_{\mathrm{k}, \text { iso }} \gtrsim$ $10^{52} \mathrm{erg}$ for every observing angle. GRB-like jets exploding in less-dense environments than we rule out will have fastermoving ejecta and thus appear to the right of the excluded phase space in this figure. This phase space associated with faster-moving ejecta is not ruled out because these jets are associated with large $E_{\mathrm{k} \text {,iso }}$ and very low densities. As the radio emission is produced in the shock front between the jet and the CSM, at low densities, the radio luminosity will be lower and more difficult to rule out, especially if it is off-axis.

\section{Constraints on Uncollimated Outflows}

Despite the fact that SLSNe-I are significantly more luminous ( 10-100 times) than "normal" Type Ic SNe at optical wavelengths, the deepest SLSN-I limits indicate that they can be significantly fainter than even some normal SNe Ic at radio wavelengths (see Figure 1). Here we analyze our radio limits in the context of uncollimated (spherical) outflows.

Among H-stripped core-collapse SNe without collimated outflows, relativistic SNe qualify as a separate class. Relativistic $\mathrm{SNe}$ are characterized by mildly relativistic ejecta, bright radio emission-but faint X-ray emission that clearly sets relativistic $\mathrm{SNe}$ apart from subenergetic GRBs-and a kinetic energy profile $E_{\mathrm{k}}(\Gamma \beta)$ (Figure 6) that is suggestive of the presence of a central engine driving the explosion (Bietenholz et al. 2010; Soderberg et al. 2010b; Chakraborti \& Ray 2011; Margutti et al. 2014; Chakraborti et al. 2015). These observed properties are attributed to a scenario where a jet is present but fails to successfully break through the stellar envelope, possibly due to a shorter-lived engine or a larger envelope mass (Lazzati et al. 2012; Margutti et al. 2014; see also Mazzali et al. 2008). To date, only two relativistic SNe, 2009bb and 2012ap, and one candidate (iPTF17cw; Corsi et al. 2017) are known. As there is 
no evidence for the beaming of the radio emission from relativistic SNe (Bietenholz et al. 2010; Soderberg et al. 2010a; Chakraborti et al. 2015), the radio limits on SLSNe-I 2017egm, Gaia16apd, and, to a lesser extent, 2012il clearly rule out the radio luminosities associated with relativistic $\mathrm{SNe}$ (Figure 1). These observations indicate some key difference of the blastwave and environment properties of SLSNe-I and relativistic $\mathrm{SNe}$ that we quantify below with simulations of the radio emission from uncollimated ejecta.

The SN shock interaction with the medium, previously sculpted by the stellar progenitor mass loss, is a well-known source of radio emission in young $\mathrm{SNe}$ (e.g., Chevalier 1982; Weiler et al. 1986; Chevalier \& Fransson 2006). The SN shock wave accelerates CSM electrons into a power-law distribution $N(\gamma) \propto \gamma^{-p}$ above a minimum Lorentz factor $\gamma_{m}$. Radio nonthermal synchrotron emission originates as the relativistic electrons gyrate in amplified magnetic fields. The result is a bell-shaped radio spectrum peaking at frequency $\nu_{p}$ and cascading down to a lower frequency as the medium becomes optically thin to SSA. In the case of H-stripped core-collapse $\mathrm{SNe}, p \sim 3$ is usually inferred, and SSA dominates over freefree absorption (e.g., Chevalier \& Fransson 2006). The selfabsorbed radio spectrum scales as $F_{\nu} \propto \nu^{5 / 2}$ below $\nu_{p}$ and $F_{\nu} \propto \nu^{-(p-1) / 2}$ above $\nu_{p}$ (Rybicki \& Lightman 1979).

We generated a grid of radio spectral models with $p=3, \nu_{p}$ between 0.1 and $60 \mathrm{GHz}$, and peak spectral luminosity $L_{\nu_{p}}$ in the range $5 \times 10^{26}-10^{29} \mathrm{erg} \mathrm{s}^{-1} \mathrm{~Hz}^{-1}$. The comparison to the SLSN-I radio limits (at all frequencies) leads to robust constraints on the time-averaged velocity of the shock wave ( $\Gamma \beta$ in Figure 1), total energy required to power the radio emission $(E)$, amplified magnetic field $(B)$, and progenitor mass-loss rate $(\dot{M})$. Our calculations assume a wind-like medium with fiducial microphysical parameters $\epsilon_{e}=0.1$ and $\epsilon_{B}=0.01$. Following Chevalier (1998), Chevalier \& Fransson (2006), and Soderberg et al. (2012), for SSA-dominated SNe, the shock-wave radius is given by

$$
\begin{aligned}
R \approx & 3.3 \times 10^{15}\left(\epsilon_{e} / \epsilon_{B}\right)^{-1 / 19}\left(L_{\nu_{p}} / 10^{26} \mathrm{erg} \mathrm{s}^{-1} \mathrm{~Hz}^{-1}\right)^{9 / 19} \\
& \times\left(\nu_{p} / 5 \mathrm{GHz}\right)^{-1} \mathrm{~cm}, \\
B \approx & 0.70\left(\epsilon_{e} / \epsilon_{B}\right)^{-4 / 19}\left(L_{\nu_{p}} / 10^{26} \mathrm{erg} \mathrm{s}^{-1} \mathrm{~Hz}^{-1}\right)^{-2 / 19} \\
& \times\left(\nu_{p} / 5 \mathrm{GHz}\right) \mathrm{G}, \\
E= & B^{2} R^{3} / 12 \epsilon_{B}, \\
\dot{M} \approx & 0.39 \times 10^{-5}\left(\epsilon_{B} / 0.1\right)^{-1}\left(\epsilon_{e} / \epsilon_{B}\right)^{-8 / 19} \\
& \times\left(L_{\nu_{p}} / 10^{26} \mathrm{erg} \mathrm{s}^{-1} \mathrm{~Hz}^{-1}\right)^{-4 / 19} \\
& \times\left(\nu_{p} / 5 \mathrm{GHz}\right)^{2} \\
& \times\left(t_{p} / 10 \text { days }\right)^{2} M_{\odot} \mathrm{yr}^{-1} .
\end{aligned}
$$

Based on these simulations, we find that the radio limits on the SLSN-I 2017egm produce interesting constraints in the $\dot{M}$, $E_{\mathrm{k}}$, and $\Gamma \beta$ phase space (orange shaded area in Figures 5 and 6 ). At any given velocity of the fastest ejecta, the limits on SN 2017 egm rule out $E_{\mathrm{k}}>10^{48} \mathrm{erg}$ coupled to the fastest ejecta (Figure 6) and the densest environments found in association with $\mathrm{H}$-stripped core-collapse $\mathrm{SNe}$ (Figure 5). Current limits, however, do not constrain the slope of the $E_{\mathrm{k}}(\Gamma \beta)$ profile or rule out the region of the parameter space populated by spherical hydrodynamical collapses with $E_{\mathrm{k}}<10^{48}$ erg in their fastest ejecta (Figure 6).

\section{Summary and Conclusions}

We have compiled all of the radio observations of SLSNe-I published to date and presented three new observations (a sample of nine SLSNe-I). Based on these limits, we constrain the subparsec environments and fastest ejecta in this sample of SLSNe-I for the case that a relativistic jet or an uncollimated outflow is present. For this analysis, we make the necessary assumption that the jet/environment properties do not vary within this sample of SLSNe-I. These are our main results.

1. In this sample of SLSNe-I, we rule out collimated on-axis jets of the kind detected in GRBs.

2. We do not rule out the entire parameter space for off-axis jets in this sample, but we do constrain the energies and circumstellar environment densities if off-axis jets are present.

3. If the SLSNe-I in this sample have off-axis GRB-like (collimated to $\theta_{\mathrm{j}}=5^{\circ}$ ) jets, then the local environment is of similar (or lower) density as that of the detected GRBs. Specifically, if off-axis jets of this kind are present, then they have energies $E_{\mathrm{k} \text {,iso }}<10^{53} \mathrm{erg}\left(E_{\mathrm{k}}<4 \times 10^{50} \mathrm{erg}\right)$ in environments shaped by progenitors with mass-loss rates $\dot{M}<10^{-4} M_{\odot} \mathrm{yr}^{-1}$ (for microphysical shock parameters $\epsilon_{e}=0.1$ and $\epsilon_{B}=0.01$ ). This would, for example, exclude jets with $E_{\mathrm{k}}>4 \times 10^{50} \mathrm{erg}$ if the progenitor mass-loss rates were of the order typically found in the winds of extreme red supergiants and luminous blue variables (and inferred for some $\mathrm{SNe} \mathrm{Ibc}$ and $\mathrm{IIb}$ ).

4. If this sample of SLSNe-I produced off-axis jets that are less collimated $\left(\theta_{\mathrm{j}}=30^{\circ}\right)$ than cosmological GRBs, then the jets must have energies $E_{\mathrm{k} \text {,iso }}<10^{53} \mathrm{erg}$ $\left(E_{\mathrm{k}}<10^{50} \mathrm{erg}\right)$ and occur in environments shaped by progenitors with mass-loss rates $\dot{M}<10^{-5} M_{\odot} \mathrm{yr}^{-1}$. This precludes jets of this kind with $E_{\mathrm{k}}<10^{50} \mathrm{erg}$ for the mass-loss rates inferred for most of the observed population of hydrogen-stripped SNe and the most dense environments inferred for GRBs.

5. The deepest SLSN-I limits rule out emission from faint uncollimated GRBs ("subluminous" or "subenergetic" GRBs), including GRB 980425 but with the exception of GRB 060218. To successfully probe emission at the level of all poorly collimated GRBs (like GRB 060218), radio observations of SLSNe-I in the local universe $(z \leqslant 0.1)$ need to be taken $\leqslant 10$ days after explosion.

6. The radio limits of this sample of SLSNe-I rule out radio luminosities associated with relativistic $\mathrm{SNe}$ and thus likely indicate some key difference of the blast-wave and environment properties between these two classes of objects. Significant differences in the respective structures of the progenitor stars and/or jet longevity may also affect the ability of the central engine's jet to successfully break through the stellar envelope.

7. We only partially rule out the phase space associated with uncollimated outflows, such as the kind seen in ordinary Type Ibc SNe.

8. For SN 2017egm (the closest SLSN-I observed at radio wavelengths to date), we can constrain the energy of a possible uncollimated outflow to $E_{\mathrm{k}} \lesssim 10^{48} \mathrm{erg}$, which is consistent with the kinetic energy associated with the fastest-moving material in ordinary Type Ibc SNe. 
Radio observations of SLSNe-I are a powerful tool to constrain the central engine properties and subparsec environments of these luminous, H-stripped stellar explosions. To fully constrain the outflow and environment properties, a combination of both early-time and late-time coverage is needed. Specifically, to probe emission at the level of all poorly collimated GRBs (like GRB 060218), radio observations of SLSNe-I in the local universe $(z \leqslant 0.1)$ need to be taken $\leqslant 10$ days after explosion. Late-time radio observations taken hundreds of days post-explosion are necessary to detect offaxis collimated jets in these systems. We will be able to constrain this much more efficiently with more sensitive radio telescopes that are coming online or planned, such as MeerKAT, the next-generation VLA (ngVLA; McKinnon et al. 2016), and the Square Kilometre Array (SKA; Carilli \& Rawlings 2004).

This research made use of the Open Supernova Catalog (Guillochon et al. 2017) and NASA's Astrophysics Data System Bibliographic Services. The National Radio Astronomy Observatory is a facility of the National Science Foundation operated under cooperative agreement by Associated Universities, Inc. CG acknowledges the University of Ferrara for use of the local HPC facility cofunded by the "Large-Scale Facilities 2010" project (grant 7746/2011). We thank the University of Ferrara and INFN-Ferrara for the access to the COKA GPU cluster. Development of the BOXFIT code was supported in part by NASA through grant NNX10AF62G issued through the Astrophysics Theory Program and by the NSF through grant AST-1009863. Simulations for BOXFIT version 2 have been carried out in part on the computing facilities of the Computational Center for Particle and Astrophysics (C2PAP) of the research cooperation "Excellence Cluster Universe" in Garching, Germany. DC and RM acknowledge partial support from program Nos. NNX16AT51G and NNX16AT81G provided by NASA through the Swift Guest Investigator Programs. Support for BAZ is based in part while serving at the NSF. At the time of the observations, LC was a Jansky Fellow of the National Radio Astronomy Observatory. GM acknowledges funding support from the UnivEarthS Labex program of Sorbonne Paris Cité (ANR-10-LABX-0023 and ANR-11-IDEX-0005-02) and from the French Research National Agency, CHAOS project ANR-12-BS05-0009.

\section{Appendix \\ Brief Description of the SLSNe-I in Our Sample}

\section{A.1. PTF09cnd}

PTF09cnd was detected by the Palomar Transient Factory during commissioning and was one of the systems that originally defined the SLSN class (Quimby et al. 2011). Properties of the host galaxy are given in Neill et al. (2011) and Perley et al. (2016). X-ray observations at $0.3-10 \mathrm{keV}$ produced nondetections with unabsorbed fluxes in the range $10^{-15}-10^{-13} \mathrm{erg} \mathrm{s}^{-1} \mathrm{~cm}^{-2}$ (Levan et al. 2013; Margutti et al. 2017a). Radio observations of PTF09cnd are reported on in Chandra et al. (2009b, 2010) (see Table 1).

\section{A.2. PS1-10awh}

PS1-10awh was discovered in 2010 October in the PanSTARRS1 Medium Deep Survey while on the rise to peak
(Chomiuk et al. 2011). UV/optical photometry and time-series spectroscopy from day -21 to 26 is presented in Chomiuk et al. (2011). Lunnan et al. (2014) detected the host galaxy at $M_{\mathrm{F} 606 \mathrm{~W}}=27$ mag with the Hubble Space Telescope. Chomiuk et al. (2011) observed PS1-10awh at radio wavelengths (see Table 1).

\section{A.3. PS1-10bzj}

PS1-10bzj was discovered in the Pan-STARRS (Chambers et al. 2016) Medium Deep Survey and classified as a SLSN-I with a peak magnitude of around -21.4 mag (Lunnan et al. 2013). Lunnan et al. found that the luminosity cannot be powered solely by radioactive nickel decay, but a magnetar or interaction model can explain the observed properties (although intermediate-width spectral lines are predicted by the interaction model, none were detected). Radio observations of this SN are presented here for the first time (see Table 2).

\section{A.4. PS1-10ky}

PS1-10ky was discovered near peak brightness in the PanSTARRS1 Medium Deep Survey at $z=0.9558$ (Chomiuk et al. 2011). At peak, its bolometric magnitude was $-22.5 \mathrm{mag}$ (see Chomiuk et al. 2011). No host has been detected for this SN (Chomiuk et al. 2011; Lunnan et al. 2015; McCrum et al. 2015). Radio observations of PS1-10ky have been presented in Chomiuk et al. (2011); see Table 1.

\section{A.5. SN 2012il}

SN 2012il (alternatively PS1-12fo or CSS120121:094613 +195028 ) was independently discovered in the Pan-STARRS1 3Pi Faint Galaxy Supernova Survey (Smartt et al. 2012) and the Catalina Real-time Transient Survey (Drake et al. 2012) on 2012 January 19 and 21, respectively. Smartt et al. (2012) classified it as an SLSN-I at $z=0.175$. Based on the late-time luminosity decline rate and the short diffusion times at peak, Inserra et al. (2013) favored the magnetar model. See Lunnan et al. (2014) for optical spectroscopy of the host galaxy. X-ray observations of SN $2012 \mathrm{il}$ in the $0.2-10 \mathrm{keV}$ band yielded nondetections with upper limits on the unabsorbed flux in the range $10^{-13}-10^{-12} \mathrm{erg} \mathrm{cm}^{-2} \mathrm{~s}^{-1}$ (Levan et al. 2013; Margutti et al. 2017b). The radio observations of this system were taken by Chomiuk et al. (2012b); see Table 1 .

\section{A.6. SN $2015 b n$}

SN 2015bn (alternatively PS15ae, CSS141223:113342 +004332 , or MLS150211:113342+004333) was discovered by the Catalina Real-time Transient Survey (Drake et al. 2009) and the Pan-STARRS Survey for Transients (Huber et al. 2015). It was classified as an SLSN-I by Le Guillou et al. (2015), which was confirmed by Drake et al. (2015). In UV (obtained with Swift) to NIR observations from -50 to 250 days from optical peak, Nicholl et al. (2016a) found that it was slowly evolving and showed large undulations (on timescales of 30-50 days) superimposed on this evolution in the light curve. The nature of these undulations is discussed in Nicholl et al. (2016a) and Yu \& Li (2017).

Nebular phase observations of SN 2015bn (Nicholl et al. 2016b; Jerkstrand et al. 2017) indicate that it is powered by a central engine (Nicholl et al. 2016b). From spectropolarimetric observations of the SN, Inserra et al. (2016) inferred an 
axisymmetric geometry and found that the evolution could be consistent with a central engine. Additional polarimetric observations can be found in Leloudas et al. (2017). X-ray observations at $0.3-10 \mathrm{keV}$ produced nondetections with unabsorbed fluxes that were predominantly in the range $10^{-14}-10^{-13} \mathrm{erg} \mathrm{s}^{-1} \mathrm{~cm}^{-2}$ (Nicholl et al. 2016a; Inserra et al. 2017; Margutti et al. 2017a). Radio observations of SN 2015bn are presented in Nicholl et al. (2016a) (see Table 1).

\section{A.7. iPTF15cyk}

The SLSN-I iPTF15cyk was detected on 2015 September 17 in optical follow-up observations of the gravitational-wave source GW150914 by the intermediate Palomar Transient Factory (Kasliwal et al. 2016). Further spectroscopic and multiwavelength observations confirmed it as an SLSN-I (Kasliwal et al. 2016). X-ray observations (Kasliwal et al. 2016) gave an upper limit on the unabsorbed flux of $1.3 \times 10^{-13} \mathrm{erg} \mathrm{cm}^{-2} \mathrm{~s}^{-1}$. Radio observations (Palliyaguru et al. 2016 and Kasliwal et al. 2016; see Table 1) were taken as part of the first broadband campaign to search for an electromagnetic counterpart to an Advanced Laser Interferometer Gravitational-Wave Observatory (LIGO) gravitationalwave trigger (Abbott et al. 2016).

\section{A.8. Gaial6apd}

Gaia16apd (alternatively SN 2016eay) was discovered by the Gaia Photometric Survey (Gaia Collaboration et al. 2016) and classified as an SLSN-I by Kangas et al. (2016). At early times, it was extremely bright at UV wavelengths (peaking at $-23.3 \mathrm{mag}$ ) in comparison to other SN classes (Blagorodnova et al. 2016; Kangas et al. 2017; Yan et al. 2017b). Based on later-time UV (observations obtained with Swift) and optical observations, Nicholl et al. (2017b) concluded that this is due to a more powerful central engine rather than interaction with the surrounding medium or a lack of UV absorption. Nicholl et al. found that the best fit for a magnetar central engine has a spin period of $2 \mathrm{~ms}$ and magnetic field of $4 \times 10^{14} \mathrm{G}$ (in agreement with Kangas et al. 2017). Radio observations of Gaia16apd are presented here for the first time (see Table 2).

\section{A.9. SN 2017egm}

SN 2017egm (alternatively Gaia17biu) was discovered by the Gaia satellite on 2017 May 26 and was originally classified as a Type II SN (Xiang et al. 2017). Dong et al. (2017) then reclassified it as an SLSN-I at $z=0.030721$, making it the closest SLSN-I discovered to date.

Although an initial X-ray detection of the SN was claimed (Grupe et al. 2017a), it was later found that the X-ray emission was more likely associated with star formation in the host galaxy, NGC 3191 (Bose et al. 2018; Grupe et al. 2017b). Several radio observations were taken at different frequencies (see Table 1 for details), but SN 2017egm was not detected (Bose et al. 2018; Bright et al. 2017; Coppejans et al. 2017; Romero-Canizales et al. 2017).

The host galaxy, NGC 3191, is unlike those of other SLSNe-I. It is a metal-rich $\left(\approx 1.3 \mathrm{Z}_{\odot}\right.$ at the radial offset of the $\left.\mathrm{SN}\right)$ massive spiral galaxy with a high star formation rate of $\approx 15 M_{\odot} \mathrm{yr}^{-1}$ and mass of $\approx 10^{10.7} M_{\odot}$ (Nicholl et al. 2017a; see also Bose et al. 2018). Izzo et al. (2018), however, showed that there are spatial variations in the host properties, and the local environment of SN 2017egm is not so unusual. NGC 3191 is also a known radio source: it was detected at $1.4 \mathrm{GHz}$ in the Faint Images of the Radio Sky at Twenty Centimeters (FIRST) survey with a peak flux density of $1.63 \pm 0.13$ mJy beam $^{-1}$ and an integrated flux density of $15.96 \pm 0.13 \mathrm{mJy}$ (White et al. 1997). Furthermore, NGC 3191 was detected at $1.8 \pm 0.1 \mathrm{mJy}$ at $15.5 \mathrm{GHz}$ (Bright et al. 2017) and detected and resolved at $10 \mathrm{GHz}$ (Bose et al. 2018). In our $33 \mathrm{GHz}$ observations, we do not detect the host galaxy to a $3 \sigma$ upper limit of $150 \mu \mathrm{Jy}_{\text {beam }^{-1}}$.

\section{ORCID iDs}

D. L. Coppejans (iD https://orcid.org/0000-0001-5126-6237

R. Margutti (i) https://orcid.org/0000-0003-4768-7586

C. Guidorzi (i) https://orcid.org/0000-0001-6869-0835

L. Chomiuk (iD https://orcid.org/0000-0002-8400-3705

K. D. Alexander (1D https://orcid.org/0000-0002-8297-2473

E. Berger (10) https://orcid.org/0000-0002-9392-9681

M. F. Bietenholz (i) https://orcid.org/0000-0002-0592-4152

W. Fong (i) https://orcid.org/0000-0002-7374-935X

A. MacFadyen (1) https://orcid.org/0000-0002-0106-9013

G. Migliori (iD https://orcid.org/0000-0003-0216-8053

D. Milisavljevic (iD https://orcid.org/0000-0002-0763-3885

M. Nicholl (ib https://orcid.org/0000-0002-2555-3192

J. T. Parrent (i) https://orcid.org/0000-0002-5103-7706

\section{References}

Abbott, B. P., Abbott, R., Abbott, T. D., et al. 2016, ApJL, 826, L13 Angus, C. R., Levan, A. J., Perley, D. A., et al. 2016, MNRAS, 458, 84 Barkat, Z., Rakavy, G., \& Sack, N. 1967, PhRvL, 18, 379

Ben-Ami, S., Gal-Yam, A., Filippenko, A. V., et al. 2012, ApJL, 760, L33 Berger, E., Kulkarni, S. R., Frail, D. A., \& Soderberg, A. M. 2003a, ApJ, 599, 408

Berger, E., Kulkarni, S. R., Pooley, G., et al. 2003b, Natur, 426, 154 Bietenholz, M. F., Soderberg, A. M., Bartel, N., et al. 2010, ApJ, 725, 4 Blagorodnova, N., Yan, L., Quimby, R., et al. 2016, ATel, 9074

Bose, S., Dong, S., Pastorello, A., et al. 2018, ApJ, 853, 57 Bright, J., Mooley, K., Fender, R., et al. 2017, ATel, 10538 Cano, Z. 2013, MNRAS, 434, 1098

Carilli, C. L., \& Rawlings, S. 2004, NewAR, 48, 979

Cenko, S. B., Frail, D. A., Harrison, F. A., et al. 2010, ApJ, 711, 641

Cenko, S. B., Frail, D. A., Harrison, F. A., et al. 2011, ApJ, 732, 29

Chakraborti, S., \& Ray, A. 2011, ApJ, 729, 57

Chakraborti, S., Soderberg, A., Chomiuk, L., et al. 2015, ApJ, 805, 187

Chambers, K. C., Magnier, E. A., Metcalfe, N., et al. 2016, arXiv:1612.05560

Chandra, P., Cenko, S. B., Frail, D. A., et al. 2008, ApJ, 683, 924

Chandra, P., Dwarkadas, V. V., Ray, A., Immler, S., \& Pooley, D. 2009a, ApJ, 699,388

Chandra, P., \& Frail, D. A. 2012, ApJ, 746, 156

Chandra, P., Ofek, E. O., Frail, D. A., et al. 2009b, ATel, 2241

Chandra, P., Ofek, E. O., Frail, D. A., et al. 2010, ATel, 2367

Chatzopoulos, E., \& Wheeler, J. C. 2012, ApJ, 760, 154

Chatzopoulos, E., Wheeler, J. C., Vinko, J., et al. 2011, ApJ, 729, 143

Chatzopoulos, E., Wheeler, J. C., Vinko, J., Horvath, Z. L., \& Nagy, A. 2013, ApJ, 773, 76

Chen, T.-W., Nicholl, M., Smartt, S. J., et al. 2017c, A\&A, 602, A9

Chen, T.-W., Schady, P., Xiao, L., et al. 2017b, ApJL, 849, L4

Chen, T.-W., Smartt, S. J., Jerkstrand, A., et al. 2015, MNRAS, 452, 1567

Chen, T.-W., Smartt, S. J., Yates, R. M., et al. 2017a, MNRAS, 470, 3566

Chevalier, R. A. 1982, ApJ, 258, 790

Chevalier, R. A. 1998, ApJ, 499, 810

Chevalier, R. A., \& Fransson, C. 2006, ApJ, 651, 381

Chevalier, R. A., \& Irwin, C. M. 2011, ApJL, 729, L6

Chevalier, R. A., \& Li, Z.-Y. 2000, ApJ, 536, 195

Chomiuk, L., Chornock, R., Soderberg, A. M., et al. 2011, ApJ, 743, 114

Chomiuk, L., Soderberg, A., Margutti, R., et al. 2012a, ATel, 3931

Chomiuk, L., Soderberg, A. M., Moe, M., et al. 2012b, ApJ, 750, 164

Coppejans, D. L., Margutti, R., Zauderer, B. A., et al. 2017, ATel, 10546 
Corsi, A., Cenko, S. B., Kasliwal, M. M., et al. 2017, ApJ, 847, 54

Corsi, A., Ofek, E. O., Frail, D. A., et al. 2011, ApJ, 741, 76

Corsi, A., Ofek, E. O., Gal-Yam, A., et al. 2014, ApJ, 782, 42

Crowther, P. A. 2007, ARA\&A, 45, 177

Curran, P. A., Evans, P. A., de Pasquale, M., Page, M. J., \& van der Horst, A. J. 2010, ApJL, 716, L135

De Cia, A., Gal-Yam, A., Rubin, A., et al. 2017, arXiv:1708.01623

de Jager, C., Nieuwenhuijzen, H., \& van der Hucht, K. A. 1988, A\&AS, 72, 259

Dessart, L., Hillier, D. J., Waldman, R., Livne, E., \& Blondin, S. 2012 MNRAS, 426, L76

Dexter, J., \& Kasen, D. 2013, ApJ, 772, 30

Dong, S., Bose, S., Chen, P., et al. 2017, ATel, 10498

Drake, A. J., Djorgovski, S. G., Mahabal, A., et al. 2009, ApJ, 696, 870

Drake, A. J., Djorgovski, S. G., Mahabal, A. A., et al. 2012, ATel, 3873

Drake, A. J., Stern, D., Djorgovski, S. G., et al. 2015, ATel, 7156

Filippenko, A. V. 1997, ARA\&A, 35, 309

Frail, D. A., Cameron, P. B., Kasliwal, M., et al. 2006, ApJL, 646, L99

Gaia Collaboration, Prusti, T., de Bruijne, J. H. J., et al. 2016, A\&A, 595, A1

Gal-Yam, A. 2012, Sci, 337, 927

Gal-Yam, A., Mazzali, P., Ofek, E. O., et al. 2009, Natur, 462, 624

Gänsicke, B. T., Levan, A. J., Marsh, T. R., \& Wheatley, P. J. 2009, ApJL, 697, L129

Ginzburg, S., \& Balberg, S. 2012, ApJ, 757, 178

Greiner, J., Mazzali, P. A., Kann, D. A., et al. 2015, Natur, 523, 189

Groh, J. H. 2014, A\&A, 572, L11

Grupe, D., Dong, S., Prieto, J. L., \& Pooley, D. 2017a, ATel, 10499

Grupe, D., Dong, S., Prieto, J. L., \& Pooley, D. 2017b, ATel, 10531

Guidorzi, C., Mundell, C. G., Harrison, R., et al. 2014, MNRAS, 438, 752

Guillochon, J., Parrent, J., Kelley, L. Z., \& Margutti, R. 2017, ApJ, 835, 64

Horesh, A., Cenko, S. B., Perley, D. A., et al. 2015, ApJ, 812, 86

Horesh, A., Kulkarni, S. R., Corsi, A., et al. 2013, ApJ, 778, 63

Huber, M., Chambers, K. C., Flewelling, H., et al. 2015, ATel, 7153

Immler, S., Wilson, A. S., \& Terashima, Y. 2002, ApJL, 573, L27

Inserra, C., Bulla, M., Sim, S. A., \& Smartt, S. J. 2016, ApJ, 831, 79

Inserra, C., Nicholl, M., Chen, T.-W., et al. 2017, MNRAS, 468, 4642

Inserra, C., Smartt, S. J., Jerkstrand, A., et al. 2013, ApJ, 770, 128

Izzo, L., Thöne, C. C., García-Benito, R., et al. 2018, A\&A, 610, A11

Jerkstrand, A., Smartt, S. J., Inserra, C., et al. 2017, ApJ, 835, 13

Kamble, A. 2015, GCN, 1, 17859

Kamble, A., Margutti, R., Soderberg, A. M., et al. 2016, ApJ, 818, 111

Kamble, A., Soderberg, A. M., Chomiuk, L., et al. 2014, ApJ, 797, 2

Kangas, T., Blagorodnova, N., Mattila, S., et al. 2017, MNRAS, 469, 1246

Kangas, T., Elias-Rosa, N., Lundqvist, P., et al. 2016, ATel, 9071

Kann, D. A., Schady, P., Olivares, E. F., et al. 2016, arXiv:1606.06791

Kasen, D., \& Bildsten, L. 2010, ApJ, 717, 245

Kasen, D., Woosley, S. E., \& Heger, A. 2011, ApJ, 734, 102

Kasliwal, M. M., Cenko, S. B., Singer, L. P., et al. 2016, ApJL, 824, L24

Kozyreva, A., Gilmer, M., Hirschi, R., et al. 2017, MNRAS, 464, 2854

Kulkarni, S. R., Frail, D. A., Wieringa, M. H., et al. 1998, Natur, 395, 663

Laskar, T., Alexander, K. D., \& Berger, E. 2016a, GCN, 1, 20328

Laskar, T., Alexander, K. D., Berger, E., et al. 2016b, ApJ, 833, 88

Laskar, T., Berger, E., Tanvir, N., et al. 2014, ApJ, 781, 1

Laskar, T., Berger, E., Zauderer, B. A., et al. 2013, ApJ, 776, 119

Lazzati, D., Morsony, B. J., Blackwell, C. H., \& Begelman, M. C. 2012, ApJ, 750, 68

Le Guillou, L., Mitra, A., Baumont, S., et al. 2015, ATel, 7102

Leloudas, G., Maund, J. R., Gal-Yam, A., et al. 2017, ApJ, 837, L14

Leloudas, G., Schulze, S., Krühler, T., et al. 2015, MNRAS, 449, 917

Levan, A. J., Read, A. M., Metzger, B. D., Wheatley, P. J., \& Tanvir, N. R. 2013, ApJ, 771, 136

Liu, L.-D., Wang, S.-Q., Wang, L.-J., et al. 2017a, ApJ, 842, 26

Liu, Y.-Q., Modjaz, M., \& Bianco, F. B. 2017b, ApJ, 845, 85

Lunnan, R., Chornock, R., Berger, E., et al. 2013, ApJ, 771, 97

Lunnan, R., Chornock, R., Berger, E., et al. 2014, ApJ, 787, 138

Lunnan, R., Chornock, R., Berger, E., et al. 2015, ApJ, 804, 90

Lunnan, R., Chornock, R., Berger, E., et al. 2016, ApJ, 831, 144

Lunnan, R., Chornock, R., Berger, E., et al. 2018, ApJ, 852, 81

Margalit, B., Metzger, B. D., Thompson, T. A., Nicholl, M., \& Sukhbold, T. 2018, MNRAS, 475, 2659

Margutti, R., Chornock, R., Metzger, B. D., et al. 2017b, arXiv:1704.05865

Margutti, R., Kamble, A., Milisavljevic, D., et al. 2017a, ApJ, 835, 140

Margutti, R., Milisavljevic, D., Soderberg, A. M., et al. 2014, ApJ, 797, 107

Margutti, R., Soderberg, A. M., Wieringa, M. H., et al. 2013b, ApJ, 778, 18

Margutti, R., Zaninoni, E., Bernardini, M. G., et al. 2013a, MNRAS, 428, 729

Massey, P., Neugent, K. F., \& Morrell, N. 2015, ApJ, 807, 81

Mazzali, P. A., Valenti, S., Della Valle, M., et al. 2008, Sci, 321, 1185
Mazzali, P. A., Walker, E. S., Pian, E., et al. 2013, MNRAS, 432, 2463

McCrum, M., Smartt, S. J., Kotak, R., et al. 2014, MNRAS, 437, 656

McCrum, M., Smartt, S. J., Rest, A., et al. 2015, MNRAS, 448, 1206

McKinnon, M., Chandler, C., Hibbard, J., Kern, J., \& Perley, M. 2016, Proc. SPIE, 9910, 99100L

McMullin, J. P., Waters, B., Schiebel, D., Young, W., \& Golap, K. 2007, in ASP Conf. Ser. 376, Astronomical Data Analysis Software and Systems XVI, ed. R. A. Shaw, F. Hill, \& D. J. Bell (San Francisco, CA: ASP), 127

Metzger, B. D., Margalit, B., Kasen, D., \& Quataert, E. 2015, MNRAS, 454, 3311

Milisavljevic, D., Margutti, R., Parrent, J. T., et al. 2015, ApJ, 799, 51

Milisavljevic, D., Soderberg, A. M., Margutti, R., et al. 2013, ApJL, 770, L38

Moriya, T. J., Blinnikov, S. I., Tominaga, N., et al. 2013, MNRAS, 428, 1020

Neill, J. D., Sullivan, M., Gal-Yam, A., et al. 2011, ApJ, 727, 15

Nicholl, M., Berger, E., Margutti, R., et al. 2016b, ApJL, 828, L18

Nicholl, M., Berger, E., Margutti, R., et al. 2017a, ApJL, 845, L8

Nicholl, M., Berger, E., Margutti, R., et al. 2017b, ApJL, 835, L8

Nicholl, M., Berger, E., Smartt, S. J., et al. 2016a, ApJ, 826, 39

Nicholl, M., Guillochon, J., \& Berger, E. 2017c, ApJ, 850, 55

Nicholl, M., Smartt, S. J., Jerkstrand, A., et al. 2013, Natur, 502, 346

Nicholl, M., Smartt, S. J., Jerkstrand, A., et al. 2014, MNRAS, 444, 2096

Ofek, E. O., Cameron, P. B., Kasliwal, M. M., et al. 2007, ApJL, 659, L13

Palliyaguru, N. T., Corsi, A., Kasliwal, M. M., et al. 2016, ApJL, 829, L28

Perley, D. A., Cenko, S. B., Corsi, A., et al. 2014, ApJ, 781, 37

Perley, D. A., Quimby, R. M., Yan, L., et al. 2016, ApJ, 830, 13

Perley, R. A., \& Butler, B. J. 2013, ApJS, 204, 19

Perley, R. A., Chandler, C. J., Butler, B. J., \& Wrobel, J. M. 2011, ApJL, 739, L1

Perna, R., Soria, R., Pooley, D., \& Stella, L. 2008, MNRAS, 384, 1638

Pooley, D., \& Lewin, W. H. G. 2004, IAUC, 8323, 2

Quimby, R. M., Kulkarni, S. R., Kasliwal, M. M., et al. 2011, Natur, 474, 487

Romero-Canizales, C., Beswick, R., Dong, S., \& Prieto, J. L. 2017, ATel, 10537

Roth, N., Kasen, D., Guillochon, J., \& Ramirez-Ruiz, E. 2016, ApJ, 827, 3

Rybicki, G. B., \& Lightman, A. P. 1979, Radiative Processes in Astrophysics (New York: Wiley-Interscience)

Sanders, N. E., Soderberg, A. M., Valenti, S., et al. 2012, ApJ, 756, 184

Santana, R., Barniol Duran, R., \& Kumar, P. 2014, ApJ, 785, 29

Schulze, S., Klose, S., Björnsson, G., et al. 2011, A\&A, 526, A23

Schulze, S., Krühler, T., Leloudas, G., et al. 2018, MNRAS, 473, 1258

Sironi, L., Keshet, U., \& Lemoine, M. 2015, SSRv, 191, 519

Smartt, S. J., Wright, D., Valenti, S., et al. 2012, ATel, 3918

Smith, N. 2014, ARA\&A, 52, 487

Smith, N., Hinkle, K. H., \& Ryde, N. 2009, AJ, 137, 3558

Smith, N., \& McCray, R. 2007, ApJL, 671, L17

Smith, N., Vink, J. S., \& de Koter, A. 2004, ApJ, 615, 475

Soderberg, A. M., Berger, E., Page, K. L., et al. 2008, Natur, 453, 469

Soderberg, A. M., Brunthaler, A., Nakar, E., Chevalier, R. A., \& Bietenholz, M. F. 2010a, ApJ, 725, 922

Soderberg, A. M., Chakraborti, S., Pignata, G., et al. 2010b, Natur, 463, 513

Soderberg, A. M., Kulkarni, S. R., Berger, E., et al. 2005, ApJ, 621, 908

Soderberg, A. M., Kulkarni, S. R., Nakar, E., et al. 2006b, Natur, 442, 1014

Soderberg, A. M., Margutti, R., Zauderer, B. A., et al. 2012, ApJ, 752, 78

Soderberg, A. M., Nakar, E., Berger, E., \& Kulkarni, S. R. 2006a, ApJ, 638,930

Soker, N., \& Gilkis, A. 2017, ApJ, 851, 95

Soria, R., Pian, E., \& Mazzali, P. A. 2004, A\&A, 413, 107

Tan, J. C., Matzner, C. D., \& McKee, C. F. 2001, ApJ, 551, 946

Terreran, G., Pumo, M. L., Chen, T.-W., et al. 2017, NatAs, 1, 713

Tolstov, A., Zhiglo, A., Nomoto, K., et al. 2017, ApJL, 845, L2

Troja, E., Sakamoto, T., Guidorzi, C., et al. 2012, ApJ, 761, 50

van der Horst, A. J. 2013, GCN, 1, 14987

van Eerten, H., van der Horst, A., \& MacFadyen, A. 2012, ApJ, 749, 44

van Loon, J. T., Cioni, M.-R. L., Zijlstra, A. A., \& Loup, C. 2005, A\&A, 438,273

Vink, J. S., \& de Koter, A. 2002, A\&A, 393, 543

Vink, J. S., \& de Koter, A. 2005, A\&A, 442, 587

Walker, E. S., Mazzali, P. A., Pian, E., et al. 2014, MNRAS, 442, 2768

Wang, S. Q., Liu, L. D., Dai, Z. G., Wang, L. J., \& Wu, X. F. 2016, ApJ, 828,87

Wang, X.-G., Zhang, B., Liang, E.-W., et al. 2015, ApJS, 219, 9

Weiler, K. W., Sramek, R. A., Panagia, N., van der Hulst, J. M., \& Salvati, M. 1986, ApJ, 301, 790

White, R. L., Becker, R. H., Helfand, D. J., \& Gregg, M. D. 1997, ApJ, 475,479 
Woosley, S. E. 2010, ApJL, 719, L204

Woosley, S. E., Blinnikov, S., \& Heger, A. 2007, Natur, 450, 390

Xiang, D., Song, H., Wang, X., et al. 2017, ATel, 10442

Xu, D., de Ugarte Postigo, A., Leloudas, G., et al. 2013, ApJ, 776, 98

Yan, L., Lunnan, R., Perley, D. A., et al. 2017b, ApJ, 848, 6
Yan, L., Quimby, R., Ofek, E., et al. 2015, ApJ, 814, 108

Yan, L., Quimby, R., Gal-Yam, A., et al. 2017a, ApJ, 840, 57

Yoshida, T., \& Umeda, H. 2011, MNRAS, 412, L78

Yu, Y.-W., \& Li, S.-Z. 2017, MNRAS, 470, 197

Zauderer, A., Berger, E., \& Laskar, T. 2012, GCN, 1, 13254 